\title{
Unperturbed hydrocarbon chains and liquid phase bilayer lipid chains: a computer simulation study
}

\author{
Alexander L. Rabinovich ${ }^{1} \cdot$ Alexander P. Lyubartsev $^{2}(1) \cdot$ Dmitrii V. Zhurkin $^{3}$
}

Received: 1 February 2017 / Revised: 13 April 2017 / Accepted: 7 June 2017 / Published online: 11 July 2017

(C) The Author(s) 2017. This article is an open access publication

\begin{abstract}
In this work, the properties of saturated and unsaturated fatty acid acyl chains 16:0, 18:0, 18:1(n-9)cis, 18:2(n-6)cis, 18:3(n-3)cis, 18:4(n-3)cis, 18:5(n-3)cis, 20:4(n-6)cis, 20:5(n-3)cis and 22:6(n-3)cis in a bilayer liquid crystalline state and similar hydrocarbon chains (with $\mathrm{CH}_{3}$ terminal groups instead of $\mathrm{C}=\mathrm{O}$ groups) in the unperturbed state characterised by a lack of long-range interaction were investigated. The unperturbed hydrocarbon chains were modelled by Monte Carlo simulations at temperature $T=303 \mathrm{~K}$; sixteen fully hydrated homogeneous liquid crystalline phosphatidylcholine bilayers containing these chains were studied by molecular dynamics simulations at the same temperature. To eliminate effects of the simulation parameters, the molecular dynamics and Monte Carlo simulations were carried out using the same structural data and force field coefficients. From these computer simulations, the average distances between terminal carbon atoms of the chains (end-to-end distances) were calculated and compared. The trends in the end-to-end distances obtained for the unperturbed chains were found to
\end{abstract}

Alexander P. Lyubartsev

alexander.lyubartsev@mmk.su.se

Alexander L. Rabinovich

rabinov@krc.karelia.ru

Dmitrii V. Zhurkin

ima-2@mail.ru

1 Institute of Biology, Karelian Research Center, Russian Academy of Sciences, Pushkinskaya 11, Petrozavodsk 185910, Russian Federation

2 Department of Materials and Environmental Chemistry, Stockholm University, 10691 Stockholm, Sweden

3 Physics and Technology Department, Petrozavodsk State University, Universitetskaya 10, Petrozavodsk 185910, Russian Federation be qualitatively similar to those obtained for the same lipid chains in the bilayers. So, for understanding of a number of processes in biological membranes (e.g., changes in fatty acid composition caused by environmental changes such as temperature and pressure), it is possible to use, at least as a first approximation, the relationships between the structure and properties for unperturbed or isolated hydrocarbon chains.

Keywords Lipid bilayers · Biomembranes · Unsaturated hydrocarbon chains $\cdot$ Molecular dynamics $\cdot$ Monte Carlo

\section{Introduction}

Biological membranes are very complex heterogeneous systems composed of various molecules such as lipids, sterols, proteins, carbohydrates, etc. Lipid molecules contain different head groups and a wide variety of acyl chains of fatty acids (FAs; Cook and McMaster 2002; Nelson and Cox 2008; Mouritsen and Bagatolli 2016). The FAs are the fundamental building blocks of all lipids in living matter. The most abundant class of lipids in the biological membranes of animals and plants is phosphatidylcholine (PC). FA acyl chains of PC lipids usually contain 12-24 carbon atoms; the most common chain lengths fall between 14 (or 16) and 22. Most of the FA acyl chains are unsaturated, containing 1-6 double bonds of the cis configuration in different positions; the majority of the double bonds in the tails are methylene-interrupted (i.e., one methylene group is localized between each pair of double bonds; Cook and McMaster 2002; Nelson and Cox 2008; Mouritsen and Bagatolli 2016). It is most common to find chains with an even number of carbon atoms, whereas odd ones are found in rare cases. 
The FA chains are often denoted in accordance with 'n-minus' nomenclature, as $N: d(n-j) c i s$, where $N$ refers to the total number of carbon atoms in the chain, $d$ is the number of the methylene-interrupted double bonds and $j$ is the position of the first double bond, counted from the methyl $\left(\mathrm{CH}_{3}\right)$ terminus of the chain, with the methyl carbon as number 1 . For brevity, below the term $(n-j)$ cis in the notation will be occasionally omitted.

It should be mentioned that unsaturated FA chains and especially polyunsaturated (PU) tails of lipids (for instance, 18:1(n-9)cis FA, 18:2(n-6)cis FA, 18:3(n-3)cis FA, 18:4(n-3)cis FA, 18:5(n-3)cis FA, 20:4(n-6)cis FA, 20:5(n-3)cis FA and 22:6(n-3)cis FA) are of great importance for the structure and function of animal and plant membranes (Table 1).
It should also be noted that all higher plants have the ability to synthesize 18:2(n-6)cis FA and 18:3(n-3)cis FA, and some can also synthesize 18:4(n-3) cis FA (Singh et al. 2005). The chains of 18:2(n-6)cis, 18:3(n-3)cis and 18:4(n-3)cis FAs accumulate in plant tissues as terminal FA metabolites (Rincón-Cervera and Guil-Guerrero 2010). 22:6(n-3)cis FA is usually the end point of 18:3(n-3)cis FA metabolism in animal tissues. On the whole, membranes that are active metabolically, as in rod outer segments, mitochondria, synaptic vesicles, etc., have high levels of PU chains.

Chemical structures of all above-mentioned $s n-2$ FA acyls and one of the PC molecules [18:0/22:6(n-3)cis PC molecule] are presented in Fig. 1.

PU FA chains have been linked to a great number of biochemical processes. One of the most notable, observations

Table 1 Occurrence of unsaturated and polyunsaturated (PU) fatty acid (FA) chains of lipids in animal and plant membranes

\begin{tabular}{|c|c|c|}
\hline Fatty acid chain & Occurrence (findings) & Refs. \\
\hline \multirow[t]{7}{*}{ 18:1(n-9)cis FA } & The most abundant monoenoic FA in plant and animal tissues & \\
\hline & $35-60 \%$ of the total FAs of peanut oil acylglycerols & Carrin and Carelli (2010) \\
\hline & $35-69 \%$ of the total FAs of peanut oil acylglycerols & Köckritz and Martin (2008) \\
\hline & $60 \%$ of the total FAs of the oil from 00-quality oilseed rape & Wittkop et al. (2009) \\
\hline & $40-70 \%$ of various vegetable oils & Pinzi et al. (2009) \\
\hline & $91-92 \%$ of HO sunflower 90 plus oil & Köckritz and Martin (2008) \\
\hline & $30-40 \%$ of the total FAs in adipose fats of animals & Nelson and Cox (2008) \\
\hline \multirow[t]{6}{*}{ 18:2(n-6)cis FA } & An ubiquitous component of plant lipids & \\
\hline & $\sim 58 \%$ in the cold-pressed black cumin seed oil & Lutterodt et al. (2010) \\
\hline & $48-74 \%$ of sunflower oil & Köckritz and Martin (2008) \\
\hline & $48-59 \%$ of soybean oil & Köckritz and Martin (2008) \\
\hline & $47-58 \%$ of cottonseed oil & Köckritz and Martin (2008) \\
\hline & $75 \%$ of the total FAs of heart cardiolipin of animals & Minkler and Hoppel (2010) \\
\hline \multirow[t]{3}{*}{ 18:3(n-3)cis FA } & Large amounts in flaxseed and walnuts & \\
\hline & $34 \%$ of the total FAs in garden cress seed oil & Diwakar et al. (2010) \\
\hline & $56-71 \%$ of the total FAs in linseed oil & Köckritz and Martin (2008) \\
\hline \multirow[t]{3}{*}{ 18:4(n-3) cis FA } & $\begin{array}{l}\text { Variable amounts in several species of fungi and animals tissues, in seeds of } \\
\text { some plant families }\end{array}$ & Guil-Guerrero (2007) \\
\hline & Up to $27 \%$ in several species of algae & Guil-Guerrero (2007) \\
\hline & Up to $18 \%$ in Echium (Boraginaceae) species & Guil-Guerrero (2007) \\
\hline 18:5(n-3)cis FA & Certain algal groups in marine phytoplankton & Napolitano et al. (1995) \\
\hline \multirow[t]{2}{*}{ 20:4(n-6)cis FA } & The mammalian cell membranes, fish oils, etc. & \\
\hline & $\begin{array}{l}30-70 \% \text { of the total FAs of triacylglycerols produced by the filamentous fungus } \\
\text { Mortierella alpina } 1 \mathrm{~S}-4\end{array}$ & Sakuradani (2010) \\
\hline 20:5(n-3)cis FA & $\begin{array}{l}\text { One of the most important FAs of the so-called '( } n-3) \text { family': animal tissues } \\
\text { (especially brain), algae, fish oils }\end{array}$ & DHA (2009) \\
\hline \multirow[t]{4}{*}{ 22:6(n-3)cis FA } & $\begin{array}{l}\text { The most unsaturated FA commonly found in nature, it regulates many cell } \\
\text { transport and synaptic functions }\end{array}$ & \\
\hline & A major constituent of fish oils, especially from tuna eyeballs & DHA (2009) \\
\hline & $40 \%$ of the PU FAs in the brain of animals & DHA (2009) \\
\hline & $60 \%$ of the PU FAs in the retina of animals & DHA (2009) \\
\hline
\end{tabular}


Fig. 1 Structures, from top to bottom, of $s n-2$ lipid chains (fatty acid acyls) of 18:1(n-9)cis, 18:2(n-6)cis, 18:3(n-3)cis, 18:4(n-3)cis, 18:5(n-3)cis, 20:4(n-6)cis and 20:5(n-3)cis; phosphatidylcholine molecule of 18:0/22:6(n-3)cis PC showing structures of $s n-2$ 22:6(n-3)cis acyl chain and $s n-1$ 18:0 acyl chain; structure of the possible $s n-1$ 16:0 acyl chain

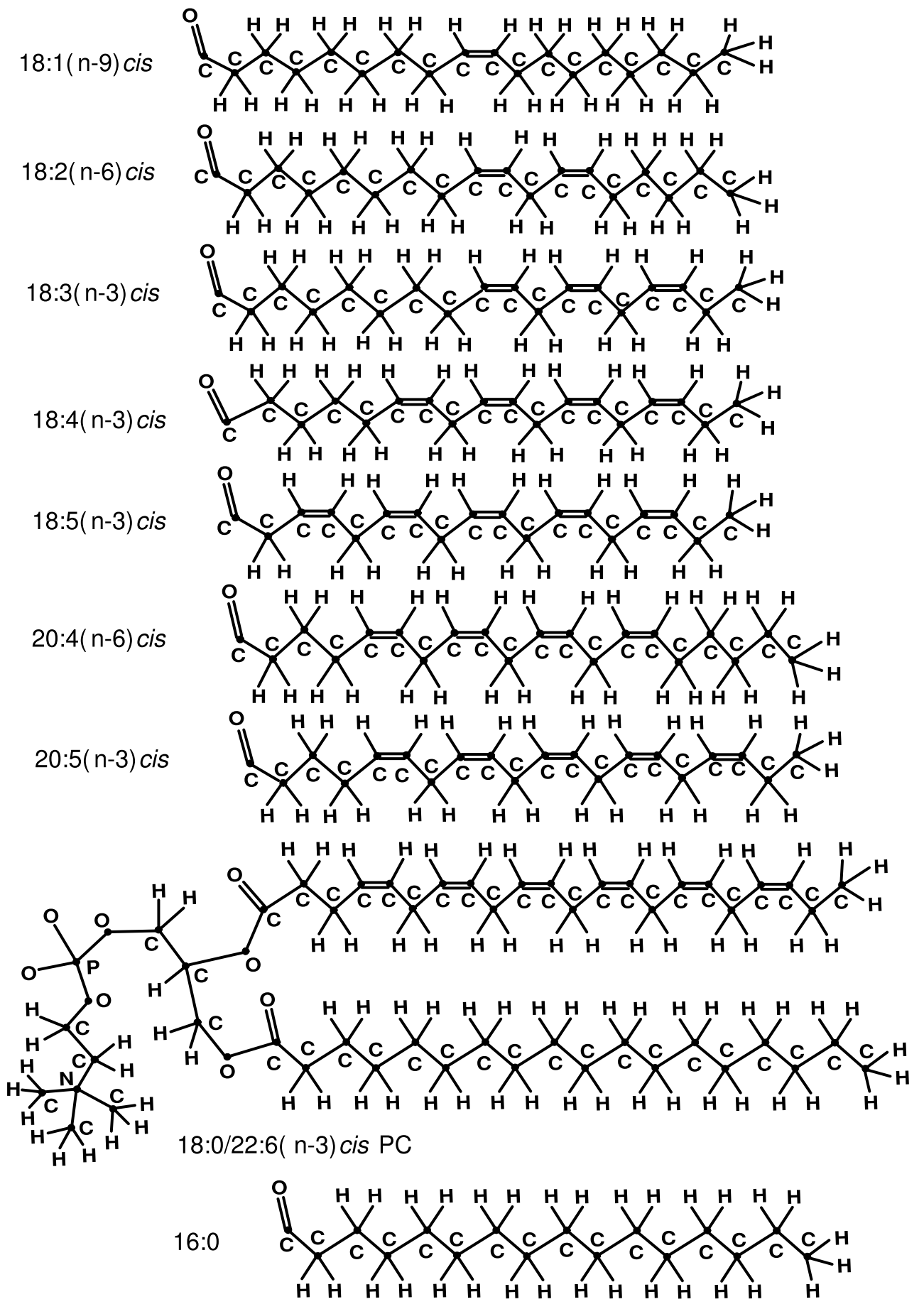

is that PU FAs play a role in achieving optimal health and in protection against disease. In other words, PU FAs (and their derivatives) have significant clinical implications. The beneficial health effects of PU FAs, particularly, 22:6(n-3)cis FA and 20:5(n-3)cis FA (DHA 2009; Sahena et al. 2009) are related to several tens of human afflictions, such as cancer (Nabavi et al. 2015; D'Eliseo and Velotti 2016; Molfino et al. 2016), cardiovascular disease
(Rovere and Christensen 2015; Sperling and Nelson 2016), allergic diseases (Rueter et al. 2015), many skin disorders (McCusker and Grant-Kels 2010), and diabetes (Bhaswant et al. 2015; Wang and Chan 2015), etc.

Thus, it is very important to study physical properties of lipid acyl chains in different conditions to reveal relationships between chemical structure and physical properties. Indeed, such relationships are of great importance 
for understanding the structure and functioning of biomembranes.

Unfortunately, experimental data for different properties of such hydrocarbons or FA acyl chains are scarce or lacking. Computer simulation is nowadays one of the most powerful tools for studying the properties of different molecular systems (Leach 2001; Berendsen 2007; Gould et al. 2007; Landau and Binder 2009; Binder and Heermann 2010; Brooks et al. 2011; Satoh 2011) including lipid membranes, lipids and lipid chains (see, e.g., several reviews on MD simulations of lipid membranes: Bennett and Tieleman 2013; Rabinovich and Lyubartsev 2013; Pluhackova and Böckmann 2015; Baoukina and Tieleman 2016; Bunker et al. 2016; Kirsch and Böckmann 2016; Lyubartsev and Rabinovich 2016; Pasenkiewicz-Gierula et al. 2016; Pöyry and Vattulainen 2016, and other articles published in Special Issue 10 of BBABiomembranes, 2016, v.1858 entitled: Biosimulations) because it allows one to obtain information on an atomistic level. On the other hand, computer simulations of lipid bilayer systems with all possible combinations of chains are still very time-consuming, and, therefore, a different approach to the task would be valuable.

To obtain 'structure-property' relationships for different hydrocarbon chains which can be compared with each other, one should use uniform conditions: the same state at the same temperature. The 'unperturbed' state of chain molecules (Flory 1969) was used as the uniform state of hydrocarbon chains in this work. The exact definition of this state is presented below, in the ' 2.2 Monte Carlo simulations' section. It has been proposed (Flory 1969) that properties of chain molecules in this state correspond to the properties in the bulk amorphous state. Neutron scattering experiments were later carried out (Dettenmaier 1978; Yoon and Flory 1978) and the results substantiated this prediction. On the other hand, biological membranes in a physiological form exist in a liquid crystalline (fluid) state having a relatively high degree of disorder and dynamical behaviour; this state is vital for the proper functioning of membranes.

The aim of the present study was to compare properties of a set of hydrocarbon chains in the unperturbed state unaffected by long-range interactions and for comparison in the liquid crystalline state of lipid bilayers, to assess if these properties are similar to each other.

A structural scheme of the chains considered in the present work is as follows: $\mathrm{CH}_{3}-\left(\mathrm{CH}_{2}\right)_{a}-\left(\mathrm{CH}=\mathrm{CH}-\mathrm{CH}_{2}\right)_{d}$ $-\left(\mathrm{CH}_{2}\right)_{b}-\mathrm{CH}_{3}$,

where $a, d, b$ are integers. The total number of carbons of the chain is $N=a+3 d+b+2$. For clear visualization of the connection between structure of the chains and FA acyl chains, these hydrocarbons will be denoted below as alk $-N: d(n-j) c i s$, i.e., similar to the 'n-minus' nomenclature for FAs.

The chains of alk-16:0, alk-18:0, alk-18:1(n-9)cis, alk-18:2(n-6)cis, alk-18:3(n-3)cis, alk-18:4(n-3)cis, alk18:5(n-3)cis, alk-20:4(n-6)cis, alk-20:5(n-3)cis and alk22:6(n-3)cis were studied by Monte Carlo (MC) simulations in an unperturbed state at temperature $T=303 \mathrm{~K}$. Chemical structures of the 10 studied hydrocarbon chains are presented in Figure 2.

In addition, 16 fully hydrated homogeneous liquid crystalline PC bilayers containing these chains (as FA acyls) were studied by molecular dynamics (MD) simulations at the same temperature $(T=303 \mathrm{~K})$. The MD and MC simulations were carried out using the same force field, to eliminate the effect of the simulation parameters. Both techniques are described below.

\section{Models and methods}

\section{Molecular dynamics simulations}

Sixteen fully hydrated homogeneous phosphatidylcholine (PC) bilayers were studied by MD simulation in an NPTensemble at temperature $T=303 \mathrm{~K}$ and pressure $P=1$ bar. The simulation software was the MDynaMix package (Lyubartsev and Laaksonen 2000). The bilayers studied were comprised of one of the PC molecules which contained a saturated $s n-1$ chain (16:0 or 18:0) and an unsaturated $s n$ - 2 chain:

1-palmitoyl-2-oleoyl-sn-glycero-3-PC $\quad[16: 0 / 18: 1(\mathrm{n}-9)$ cis $\mathrm{PC}]$

1-stearoyl-2-oleoyl-sn-glycero-3-PC $\quad[18: 0 / 18: 1(\mathrm{n}-9)$ cis $\mathrm{PC}]$ $\mathrm{PC}]$;

1-palmitoyl-2-linoleoyl-sn-glycero-3-PC [16:0/18:2(n-6)cis

1-stearoyl-2-linoleoyl-sn-glycero-3-PC [18:0/18:2(n-6)cis $\mathrm{PC}]$

1 -palmitoyl-2-linolenoyl-sn-glycero-3-PC [16:0/18:3(n-3) cis PC];

1-stearoyl-2-linolenoyl-sn-glycero-3-PC [18:0/18:3(n-3)cis $\mathrm{PC}$;

1-palmitoyl-2-octadecatetraenoyl-sn-glycero-3-PC [16:0/18:4(n-3)cis PC];

1-stearoyl-2-octadecatetraenoyl-sn-glycero-3-PC [18:0/18:4(n-3)cis PC];

1-palmitoyl-2-octadecapentaenoyl-sn-glycero-3-PC [16:0/18:5(n-3) cis PC];

1-stearoyl-2-octadecapentaenoyl-sn-glycero-3-PC [18:0/18:5(n-3) cis PC];

1-palmitoyl-2-arahidonoyl-sn-glycero-3-PC [16:0/20:4(n-6) cis PC]; 
Fig. 2 Structures, from top to bottom, of hydrocarbon chains alk-16:0, alk-18:0, alk-18:1(n9)cis, alk-18:2(n-6)cis, alk-

18:3(n-3)cis, alk-18:4(n-3)cis, alk-18:5(n-3)cis, alk-20:4(n6)cis, alk-20:5(n-3)cis and alk22:6(n-3) cis studied by Monte Carlo simulations. Such names of the hydrocarbon chains are used to stress the chain and corresponding FA acyl (Fig. 1) structural similarity alk-16:0

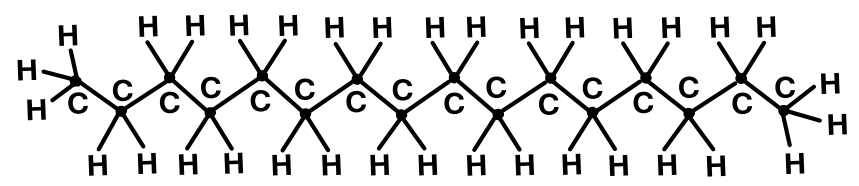

alk-18:0

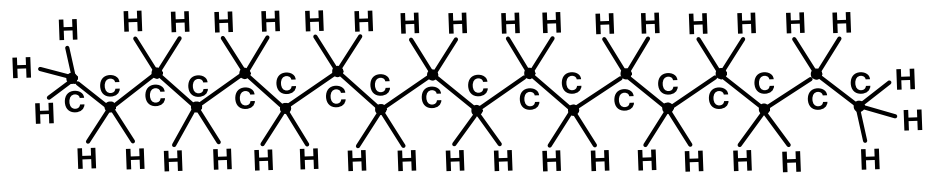

alk-18:1(n-9) cis

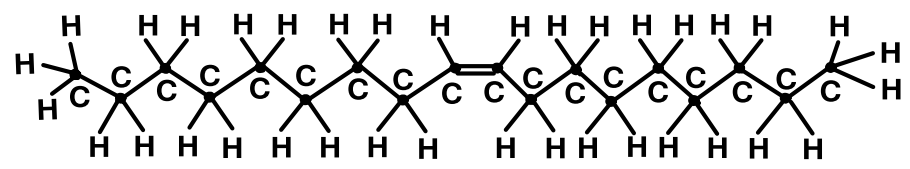

alk-18:2(n-6) cis

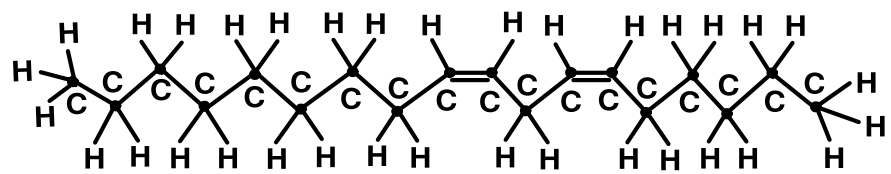

alk-18:3(n-3) cis

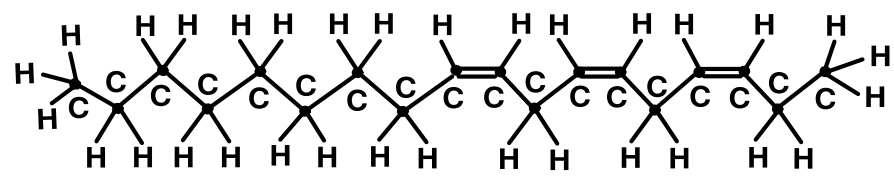

alk-18:4(n-3) cis

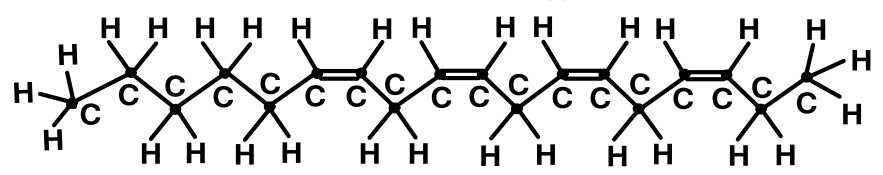

alk-18:5(n-3) cis

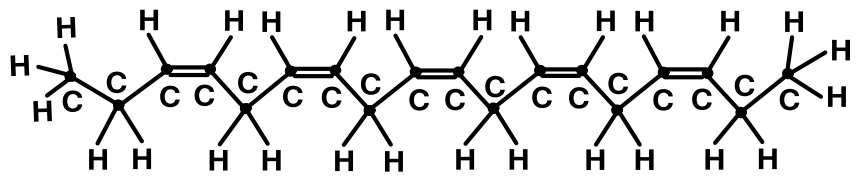

alk-20:4(n-6) cis

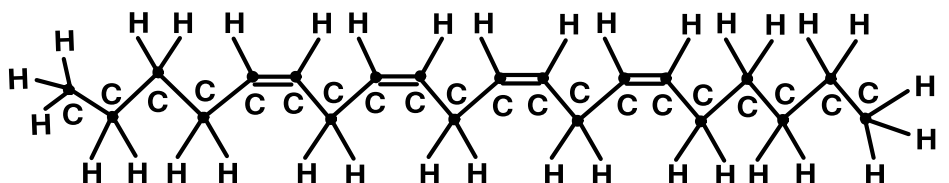

alk-20:5(n-3) cis

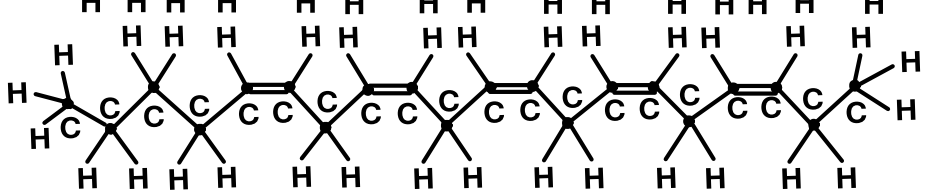

alk-22:6(n-3) cis

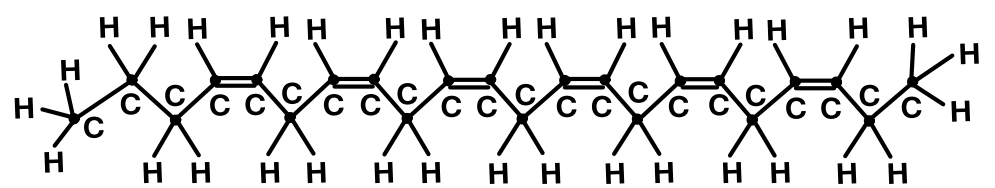

1 -stearoyl-2-arahidonoyl-sn-glycero-3-PC [18:0/20:4(n-6)cis PC];

1-palmitoyl-2-eicosapentaenoyl-sn-glycero-3-PC [16:0/20:5(n-3)cis PC];

1-stearoyl-2-eicosapentaenoyl-sn-glycero-3-PC [18:0/20:5(n-3) cis PC];

1-palmitoyl-2-docosahexaenoyl-sn-glycero-3-PC [16:0/22:6(n-3)cis PC];

1-stearoyl-2-docosahexaenoyl-sn-glycero-3-PC [18:0/22:6(n-3)cis PC].
The choice of the lipid set was motivated by the following considerations: (1) the sixteen bilayers listed contain the most important, biologically meaningful types of PU lipids (see "Introduction" section); (2) An inspection of these bilayers under the same conditions allows one to study:

1. the 'double bond number dependence' (' $d$ dependence') of an $s n-2$ chain of lipid properties over the whole possible range of $d$ from 1 to 5 at fixed $N=18$ 
Table 2 Published temperatures $T_{c}$ of the experimental lamellar gel to liquid-crystalline phase transition of phosphatidylcholines 16:0/18:1(n-9)cis PC

\begin{tabular}{llll}
\hline Lipid & $T_{c}(K)$ & Method & Refs. \\
\hline 16:0/18:1(n-9)cis PC & $266.15^{\text {a }}$ & Raman spectr. & Lavialle and Levin (1980) \\
& 268.15 & DSC & Kruyff et al. (1973) \\
268.15 & ${ }^{2}$ H NMR & Waespe-Sarcevic (1978 \\
& & & Perly et al. (1985) and Ghosh (1988) \\
& 268.15 & SANS & Winter and Pilgrim (1989) \\
$268.55 \pm 0.07^{\mathrm{b}}$ & DSC & Tada et al. (2009) \\
268.55 & DSC & Tada et al. (2010) \\
268.65 & DSC & Santaren et al. (1982) \\
270.15 & DSC & Davis et al. (1980) and Dekker et al. (1983) \\
& & Curatolo et al. (1985) \\
& & Lynch and Steponkus (1989) \\
$270.55 \pm 0.2$ & DSC & Davis et al. (1981) and Keough (1986) \\
270.65 & DSC & Swaney (1980) \\
$270.65^{\mathrm{a}}$ & Raman spectr. & Lavialle and Levin (1980) \\
270.65 & Raman spectr. & Litman et al. (1991) \\
$270.65 \pm 0.2$ & DSC & Hernandez-Borrell and Keough (1993) \\
271.15 & DSC & Curatolo (1985) and Curatolo (1986) \\
271.55 & DSC & Ichimori et al. (1999) \\
$271.65^{\mathrm{c}}$ & Raman spectr. & Lavialle and Levin (1980) \\
$272.95 \pm 0.77$ & DSC & Bryant et al. (1992) \\
276.15 & Hydrol. meas. & Kamp et al. (1975) \\
$270.65 \pm 2.4$ & lipid database & Koynova and Caffrey (1998) \\
\hline
\end{tabular}

$D S C$ differential scanning calorimetry, ${ }^{2} H$ NMR deuterium nuclear magnetic resonance, SANS small-angle neutron scattering, Hydrol. meas. hydrolysis measure. $T_{c}$ is the temperature averaged over the gel to liquidcrystalline and liquid-crystalline to gel phase transition temperatures, i.e., the heating and cooling transition temperatures, in all cases when a hysteresis was observed. The $T_{c}$ values for each lipid are presented in order of increasing temperature; values from the lipid database (Koynova and Caffrey 1998) are also presented in the end of the experimental data list.

${ }^{a}$ The existence of metastable forms for the pure 16:0/18:1(n-9)cis PC liposomes was detected in Ref. Lavialle and Levin (1980). The $I_{2940} / I_{2885}$ peak height intensity ratio as index was used (interchain disorderorder parameter), where $I_{2940}$ and $I_{2885}$ represent the peak height intensities for 2940- and $2885-\mathrm{cm}^{-1}$ transitions.

${ }^{\mathrm{b}}$ Aqueous $50 \mathrm{wt} \%$ ethylene glycol solution.

${ }^{\mathrm{c}}$ The $I_{1100} / I_{1130}$ peak height intensity ratio as index was used (intramolecular gauche-trans isomerization parameter), where $I_{1100}$ and $I_{1130}$ represent the peak height intensities for 1100 - and $1130-\mathrm{cm}^{-1}$ transitions, respectively in a sequence of $18: 1,18: 2,18: 3,18: 4$ and 18:5 chains with methylene-interrupted double bonds, all other factors being equal;

2. the effect of $s n-1$ chain elongation (from 16 to 18 carbons) on the lipid properties for different fixed $s n-2$ chains, all other factors being equal;

3. the effect of $s n-2$ chain elongation (from 18 to 20 carbons) on the lipid properties for different fixed $s n-1$ chains and fixed double bond number $d=4$ or 5 of $s n-2$ chain, all other factors being equal.

The presence of the acyl chain 16:0 in the 8 bilayers $16: 0 / \ldots$ PC and acyl chain 18:0 in the 8 bilayers $18: 0 / . . P C$ is sufficient to study geometrical properties of acyls 16:0 and 18:0 by MD simulation. End-to-end distances (between carbons) calculated for each saturated acyl were averaged over eight corresponding PC bilayers; the influence of the position $(s n-1$ instead of $s n-2)$ of these chains on their end-to-end distances was neglected.

Experimental melting temperatures $T_{c}$ of the bilayers were checked to make sure that the temperature $T=303 \mathrm{~K}$ in the MD simulations was appropriate. Available published experimental temperatures $T_{c}$ for the lamellar gel to liquidcrystalline phase transition of five of eight studied mixedchain PCs with 16:0 chains in the $s n-1$ position are gathered in Tables 2 and 3, and six of eight simulated PCs with 18:0 $s n$-1-chains are gathered in Tables 4 and 5. To the authors' knowledge, no experimental investigations of the melting 
Table 3 Published temperatures $T_{c}$ of the experimental lamellar gel to liquid-crystalline phase transition of mixed-chain phosphatidylcholines containing an $s n$-1 palmitoyl (16:0) chain and various $s n-2$ unsaturated fatty acid chains

\begin{tabular}{llll}
\hline Lipid & $T_{c}(K)$ & Method & Refs. \\
\hline 16:0/18:2(n-6)cis PC & 253.15 & DSC & Lynch and Steponkus (1989) \\
& 253.65 & DSC & Keough et al. (1989) \\
& 254.45 & DSC & Hernandez-Borrell and Keough (1993) \\
& $253.55 \pm 0.4$ & Lipid database & Koynova and Caffrey (1998) \\
16:0/18:3(n-3)cis PC & $258.15 \pm 0.5$ & ${ }^{2}$ H NMR & McCabe et al. (1994) \\
16:0/20:4(n-6)cis PC & 250.65 & Raman spectr. & Litman et al. (1991) \\
& $252.55 \pm 0.2$ & DSC & Hernandez-Borrell and Keough (1993) \\
& 250.65 & Lipid database & Koynova and Caffrey (1998) \\
16:0/22:6(n-3)cis PC & 261.85 & DSC & Hernandez-Borrell and Keough (1993) \\
& $265.3 \pm 0.5$ & ${ }^{2}$ H NMR & Barry et al. (1991) \\
& 266.65 & ${ }^{2}$ H NMR & Deese et al. (1981) \\
& 270.15 & Raman spectr. & Litman et al. (1991) \\
& 270.15 & Lipid database & Koynova and Caffrey (1998) \\
\hline
\end{tabular}

For abbreviations, see footnote in Table 2

Table 4 Published temperatures $T_{c}$ of the experimental lamellar gel to liquid-crystalline phase transition of phosphatidylcholines 16:0/18:1(n-9)cis PC

\begin{tabular}{|c|c|c|c|}
\hline Lipid & $T_{c}(K)$ & Method & Refs. \\
\hline \multirow[t]{23}{*}{ 18:0/18:1(n-9)cis PC } & $272.0 \pm 0.2$ & DSC & Niebylski and Jr. (1994) \\
\hline & 275.15 & Fluor. anis. & Vincent et al. (1985) \\
\hline & 276.15 & DSC & Phillips et al. (1972) \\
\hline & $277.25 \pm 0.1$ & DSC & Sánchez-Migallón et al. (1996) \\
\hline & $277.95 \pm 0.5$ & ${ }^{2} \mathrm{H}$ NMR & Holte et al. (1995) \\
\hline & $277.95 \pm 0.08^{\mathrm{a}}$ & DSC & Tada et al. (2009) \\
\hline & 278.20 & DSC & Lüscher-Mattli (1989) \\
\hline & 278.45 & DSC & Cunningham et al. (1989) \\
\hline & 278.75 & DSC & Wang et al. (1995b, 1995a) and Huang et al. (1996) \\
\hline & 278.85 & DSC & Inoue et al. (1999) \\
\hline & 279.05 & DSC & Stillwell et al. (2000) \\
\hline & 279.15 & DSC & Vilchéze et al. (1996) \\
\hline & 279.35 & DSC & Surewicz and Epand (1986) \\
\hline & $279.45 \pm 0.4$ & DSC & Davis et al. $(1980,1981)$ and Keough (1986) \\
\hline & 279.55 & DSC & Davis et al. (1986) \\
\hline & 279.75 & DSC & Boggs and Tümmler (1993) \\
\hline & 279.85 & DSC & Kaneshina et al. (1998) and Ichimori et al. (1999) \\
\hline & & & Broniec et al. (2009) and Tada et al. (2010) \\
\hline & $279.85 \pm 0.1^{\mathrm{b}}$ & DSC & Tada et al. (2009) \\
\hline & 280.55 & DSC & Davis and Keough (1983) \\
\hline & 281.35 & DSC & Dai et al. (1991) \\
\hline & 286.15 & Hydrol. meas. & Kamp et al. (1975) \\
\hline & $280.05 \pm 2.9$ & Lipid database & Koynova and Caffrey (1998) \\
\hline
\end{tabular}

For abbreviations, see footnote in Table 2; fluor. anis. = fluorescence anisotropy

${ }^{a}$ Aqueous $50 \mathrm{wt} \%$ ethylene glycol solution

${ }^{\mathrm{b}}$ Solvent: water

temperatures of 16:0/18:4(n-3)cis PC, 16:0/18:5(n-3)cis PC, 16:0/20:5(n-3)cis PC, 18:0/18:4(n-3)cis PC or 18:0/18:5(n-3) cis $\mathrm{PC}$ have been published.
It is seen that the temperature $T=303 \mathrm{~K}$ of the MD computer simulations is higher than experimental gel to liquid-crystalline phase transition temperatures $T_{c}$ 
Table 5 Published temperatures $T_{c}$ of the experimental lamellar gel to liquid-crystalline phase transition of mixed-chain phosphatidylcholines containing an $s n$-1 stearoyl (18:0) chain and various $s n-2$ unsaturated fatty acid chains

\begin{tabular}{|c|c|c|c|}
\hline Lipid & $T_{c}(K)$ & Method & Ref. \\
\hline \multirow[t]{8}{*}{ 18:0/18:2(n-6)cis $\mathrm{PC}$} & $256.45 \pm 0.3$ & DSC & Sánchez-Migallón et al. (1996) \\
\hline & 256.65 & DSC & Coolbear and Keough (1983) \\
\hline & $256.95 \pm 1.6$ & DSC & Coolbear et al. (1983) \\
\hline & $257.95 \pm 0.1$ & DSC & Niebylski and Jr. (1994) \\
\hline & $257.95 \pm 0.5$ & ${ }^{2} \mathrm{H}$ NMR & Holte et al. (1995) \\
\hline & 258.15 & DSC & Keough and Parsons (1990) and Tada et al. (2010) \\
\hline & 259.45 & DSC & Keough et al. (1989) \\
\hline & $258.75 \pm 4.1$ & Lipid database & Koynova and Caffrey (1998) \\
\hline \multirow[t]{6}{*}{ 18:0/18:3(n-3)cis PC } & $259.25 \pm 0.2$ & DSC & Sánchez-Migallón et al. (1996) \\
\hline & 260.15 & DSC & Coolbear et al. (1983) \\
\hline & $260.25 \pm 0.3$ & DSC & Niebylski and Jr. (1994) \\
\hline & 261.15 & DSC & Coolbear and Keough (1983) \\
\hline & $261.25 \pm 0.5$ & ${ }^{2} \mathrm{H}$ NMR & Holte et al. (1995) \\
\hline & $260.85 \pm 1.2$ & Lipid database & Koynova and Caffrey (1998) \\
\hline \multirow[t]{6}{*}{ 18:0/20:4(n-6)cis PC } & $257.55 \pm 0.3$ & DSC & Sánchez-Migallón et al. (1996) \\
\hline & $258.45 \pm 1.0$ & DSC & Niebylski and Jr. (1994) \\
\hline & $259.65 \pm 0.5$ & ${ }^{2} \mathrm{H}$ NMR & Holte et al. (1995) \\
\hline & 260.15 & DSC & Ichimori et al. (1999) and Tada et al. (2010) \\
\hline & $260.55 \pm 1.0$ & DSC & Coolbear et al. (1983) \\
\hline & $260.25 \pm 0.4$ & Lipid database & Koynova and Caffrey (1998) \\
\hline \multirow[t]{3}{*}{ 18:0/20:5(n-3)cis PC } & $260.55 \pm 0.5$ & ${ }^{2} \mathrm{H}$ NMR & Holte et al. (1995) \\
\hline & $262.05 \pm 0.1$ & DSC & Niebylski and Jr. (1994) \\
\hline & $262.75 \pm 0.1$ & Lipid database & Koynova and Caffrey (1998) \\
\hline \multirow[t]{7}{*}{ 18:0/22:6(n-3)cis PC } & 263.95 & DSC & Dumaual et al. (2000) and Stillwell et al. (2000) \\
\hline & 264.15 & DSC & Tada et al. (2010) \\
\hline & $265.60 \pm 0.5$ & ${ }^{2} \mathrm{H}$ NMR & Holte et al. (1995) \\
\hline & 265.95 & DSC & Ichimori et al. (1999) \\
\hline & $266.55 \pm 0.3$ & DSC & Niebylski and Jr. (1994) \\
\hline & $266.65 \pm 0.7$ & ${ }^{2} \mathrm{H}$ NMR & Barry et al. (1991) \\
\hline & $269.35 \pm 1.8$ & Lipid database & Koynova and Caffrey (1998) \\
\hline
\end{tabular}

For abbreviations, see footnote in Table 2 of all the bilayers in Tables 2, 3, 4 and 5. In spite of the fact that $T_{c}$ values of several lipids, 16:0/18:4(n-3)cis PC, 16:0/18:5(n-3)cis PC, 16:0/20:5(n-3)cis PC, 18:0/18:4(n-3)cis PC and 16:0/18:5(n-3)cis PC, are unknown, there is good reason to believe from the analysis of the noted Tables that missing values of $T_{c}$ are also less than $T=303 \mathrm{~K}$. This temperature is acceptable also for MC simulations of hydrocarbon chains because the main phase transition temperature of octadecane (alk-18:0) is $301.2 \mathrm{~K}$ and that of hexadecane (alk-16:0) is $291.2 \mathrm{~K}$ (Dirand et al. 2002), and phase transition temperatures of unsaturated (alkene) chains are substantially lower than those of n-alkanes.

A description of the MD simulations technique of PC bilayers was presented in a previous paper (Rabinovich and Lyubartsev 2014). The simulation boxes contained 128 PC molecules of one of 16 studied types per bilayer (64 lipids in each leaflet) and $30 \mathrm{H}_{2} \mathrm{O}$ molecules per lipid that corresponds to a condition of full hydration (overall 3840 water molecules). The two hydrocarbon tails, the glycerol section and the head group of the lipid molecules were treated in accordance with their known chemical structure, all hydrogen atoms were explicitly included in the computations.

In the starting configuration, the lipids were set parallel to each other, organized in a regular manner in two layers, and water molecules were distributed outside the bilayer. The system was put into a rectangular periodic cell, with the $Z$ axis parallel to the bilayer normal. The size of the box was varied during the simulations under a semianisotropic NPT-ensemble with two degrees of freedom: one in the $Z$ direction and another in the $X Y$ direction, so that the box sizes in $X$ and $Y$ direction were equal at each time moment. 
Fig. 3 Time evolution of the average area per lipid $A_{\mathrm{pl}}$ of the PC bilayers with $s n-1$ chain 16:0 (a) and 18:0 (b)
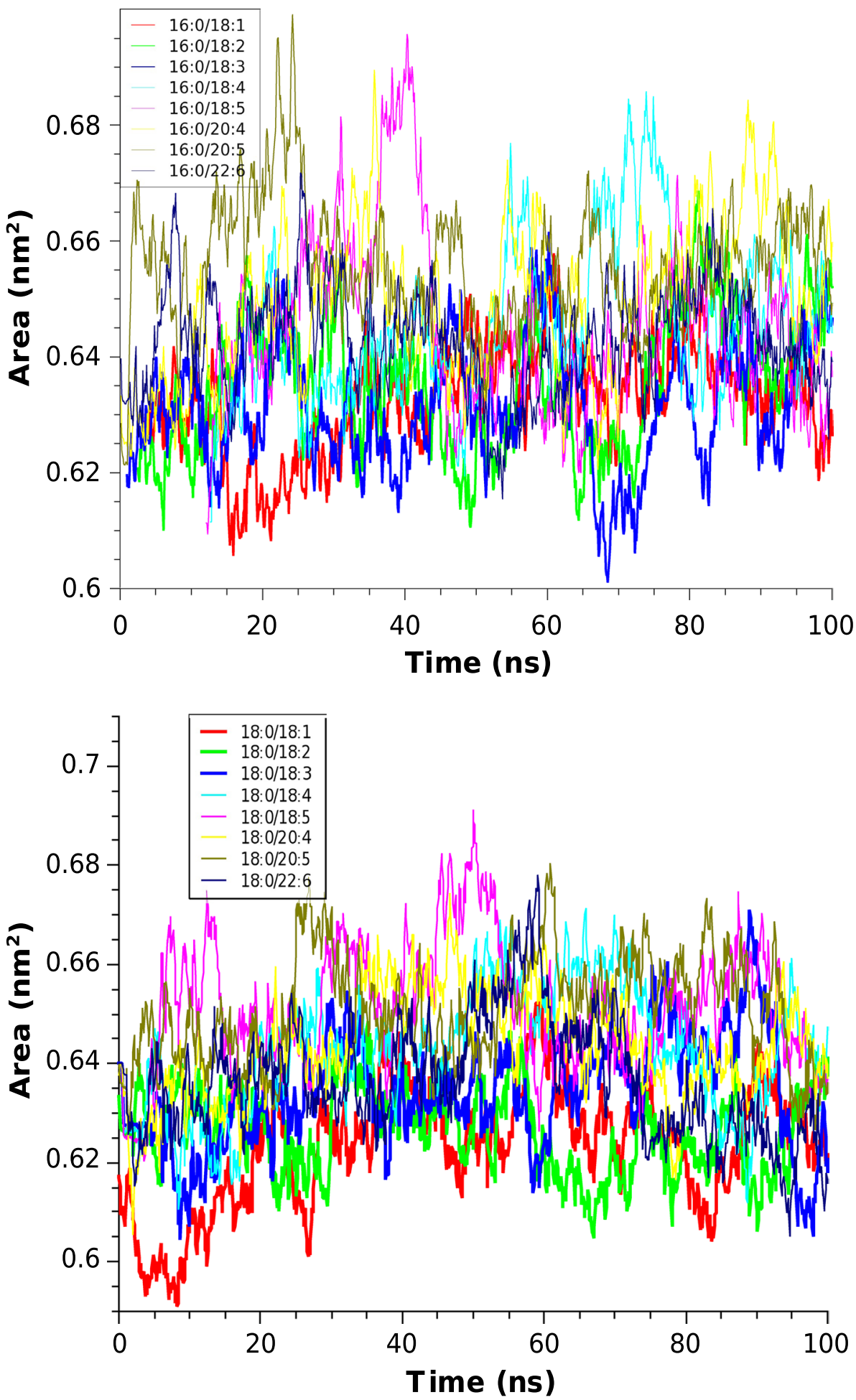

To calculate the energy of the lipid molecules in the course of MD simulations, the CHARMM27 force field parameter set (Feller and MacKerell, Jr. 2000) with modifications described in a previous paper (Högberg et al. 2008) was used. It was demonstrated in a number of publications that the original CHARMM27 force field has some disagreements with the experiment (Benz et al. 2005), especially in the tensionless isothermal-isobaric (NPT) ensemble simulations (Hyvönen and Kovanen 2005; Sonne et al. 2007). CHARMM27 force field was recommended to 
apply only with a fixed surface area; otherwise the simulated bilayer tends to form a gel-like state (Feller et al. 2002; Koubi et al. 2003; Jensen et al. 2004; Siu et al. 2008). For bilayers composed of 14:0/14:0 PC lipids, modifications introduced previously (Högberg et al. 2008) provided perfect agreement with experimental data for the area per lipid, as well as with the X-ray structure factor and NMR order parameters. For lipids considered in this work, we used the same partial charges as in Högberg et al. 2008, the lipid head group including esters (these charges were recalculated on the bases of ab-initio computations), while for tails, we used charges adopted from the original CHARMM27 force field (Feller and MacKerell, Jr. 2000), with scaling of 1-4 electrostatic interactions by factor 0.83 (Högberg et al. 2008). All intramolecular bond and angle parameters, as well as Lennard-Jones interactions, were also borrowed from the original CHARMM27 force field (Feller and MacKerell, Jr. 2000). Water molecules were described by the flexible SPC model (Toukan and Rahman 1985). Use of this water model, in connection with the modified CHARMM27 force field, was verified previously (Högberg et al. 2008).

The double time step algorithm (Tuckerman et al. 1992) was used to treat separately fast forces (covalent bonds, angles, torsions, collision Lennard-Jones forces within 5 $\AA$ A distance) with time step $0.25 \mathrm{fs}$, and longer range forces with time step 2.5 fs. The long-range electrostatic interactions were treated by the Ewald summation method (Allen and Tildesley 1987). The reciprocal part of the Ewald sum was cut at the condition that the remaining terms do not contribute more than 0.0001 of the total value. The $\alpha$ parameter of the Ewald sum was set to $\alpha=2.6 r_{\text {cut }}$, and cutoff distance $r_{\text {cut }}=14 \AA$ was optimized for computational performance according to Ref. (Fincham 1994). The dispersion correction from the Lennard-Jones interactions outside the cut-off distance was included in the pressure (Allen and Tildesley 1987).

The systems were firstly simulated $1 \mathrm{~ns}$ under constant volume and then $1 \mathrm{~ns}$ under constant pressure and isotropic cell fluctuations. The obtained configurations were considered as starting points for longer simulations with independent cell fluctuations in $\mathrm{Z}$ and $\mathrm{XY}$ directions. The time reversible Nose-Hoover constant-temperature-constantpressure algorithm (Martyna et al. 1996) was implemented, with the thermostat and barostat relaxation time set to $30 \mathrm{fs}$ and $1 \mathrm{ps}$, respectively.

All the PC bilayers were simulated for a total of $100 \mathrm{~ns}$. One of the most fundamental properties of a lipid bilayer and one of the most common ways to determine whether the bilayer system has reached equilibrium is area per lipid $A_{\mathrm{pl}}$. When the area per lipid reaches a stable value, other structural properties (density distributions, NMR order parameters) do not show noticeable trends either. In the present work, area per lipid $A_{\mathrm{pl}}$ was calculated as the crosssectional area of the simulation box divided by the number of lipids per monolayer. The time evolution of the $A_{\mathrm{pl}}$ values of the each of 16 PC bilayers are shown in Figs. 3a, b.

From the observation of the different time evolution traces and calculation of block averages, we concluded that $20 \mathrm{~ns}$ of equilibration time is enough for all the bilayer systems considered. Therefore, the first $20 \mathrm{~ns}$ of the simulations (from $100 \mathrm{~ns}$ ) were disregarded in further analysis. Atomic coordinates were saved each $1 \mathrm{ps}$ in the trajectories.

The average areas $A_{\mathrm{pl}}$ calculated as a result of our simulations are presented in Table 6.

It is possible to compare our data with the available published (experimental) data: in Table 7, available experimental average lipid areas for bilayers formed by PC lipids with one fully saturated and one unsaturated chain are collected. It is seen that our results are in good agreement with the experimentally deduced data.

\section{Monte Carlo simulations}

According to concepts developed by Flory (1969), the interpretation of the spatial configuration of a linear chain molecule dispersed in a dilute solution can be resolved into two parts: short-range and long-range interactions. (1) The short-range interactions of the chain are determined by the

Table 6 Average areas per lipid, $A_{\mathrm{pl}}$, and relative fluctuations of the areas obtained for mixed-chain liquid-crystalline phase unsaturated phosphatidylcholine bilayers by MD simulations of the present work; $T=303 \mathrm{~K}$

\begin{tabular}{lll}
\hline Lipid & $A_{\mathrm{pl}}( \pm \Delta)^{\mathrm{a}}, \mathrm{nm}^{2}$ & Rel. fluct. of area \\
\hline 16:0/18:1(n-9)cis PC & $0.634( \pm 0.002)$ & 0.01436 \\
16:0/18:2(n-6)cis PC & $0.636( \pm 0.003)$ & 0.01839 \\
16:0/18:3(n-3)cis PC & $0.632( \pm 0.002)$ & 0.01835 \\
16:0/18:4(n-3)cis PC & $0.647( \pm 0.003)$ & 0.02087 \\
16:0/18:5(n-3)cis PC & $0.646( \pm 0.004)$ & 0.02369 \\
16:0/20:4(n-6)cis PC & $0.652( \pm 0.003)$ & 0.01932 \\
16:0/20:5(n-3)cis PC & $0.656( \pm 0.003)$ & 0.01602 \\
16:0/22:6(n-3)cis PC & $0.643( \pm 0.002)$ & 0.01446 \\
18:0/18:1(n-9)cis PC & $0.627( \pm 0.002)$ & 0.01515 \\
18:0/18:2(n-6)cis PC & $0.625( \pm 0.002)$ & 0.01503 \\
18:0/18:3(n-3)cis PC & $0.637( \pm 0.002)$ & 0.01790 \\
18:0/18:4(n-3)cis PC & $0.646( \pm 0.003)$ & 0.01702 \\
18:0/18:5(n-3)cis PC & $0.653( \pm 0.003)$ & 0.01915 \\
18:0/20:4(n-6)cis PC & $0.646( \pm 0.003)$ & 0.01516 \\
18:0/20:5(n-3)cis PC & $0.653( \pm 0.002)$ & 0.01685 \\
18:0/22:6(n-3)cis PC & $0.637( \pm 0.004)$ & 0.02024 \\
\hline
\end{tabular}

a Statistical error $\Delta$ for $20-100 \mathrm{~ns}$ is evaluated from the variance of 10-ns block averages

${ }^{\mathrm{b}}\left(\left\langle\left(A_{\mathrm{pl}}-\left\langle A_{\mathrm{pl}}\right\rangle\right)^{2}\right\rangle\right)^{1 / 2} /\left\langle A_{\mathrm{pl}}\right\rangle$ 
Table 7 Published experimental average areas per lipid, $A_{\mathrm{pl}}$, of liquid-crystalline phase mixed-chain PC bilayers ( ${ }^{\mathrm{a} e s t i m a t e d ~ f r o m ~ a ~ p l o t) ~}$

\begin{tabular}{|c|c|c|c|c|}
\hline Lipid & $T(\mathrm{~K})$ & $A_{\mathrm{pl}}\left(n m^{2}\right)$ & Method & Refs. \\
\hline \multirow[t]{18}{*}{ 16:0/18:1(n-9)cis PC } & 275 & $0.54 \pm 0.01$ & $\mathrm{X}$-ray scattering & Pabst et al. (2000) \\
\hline & 293 & $0.627 \pm 0.013$ & $\mathrm{X}$-ray scattering & Kučerka et al. (2011) \\
\hline & 297 & 0.63 & Langmuir film balance & Smaby et al. (1997) \\
\hline & \multirow[t]{2}{*}{298} & \multirow[t]{2}{*}{$0.64 \pm 0.01$} & \multirow[t]{2}{*}{ Isopiestic method } & Klose et al. (1992) \\
\hline & & & & Köenig et al. (1997a) \\
\hline & 298 & $0.65 \pm 0.03$ & Fluoresc. reson. energy transf. & Lantzsch et al. (1994) and Lantzsch et al. (1996) \\
\hline & \multirow[t]{2}{*}{298} & \multirow[t]{2}{*}{$0.66 \pm 0.02$} & \multirow[t]{2}{*}{$\mathrm{X}$-ray diffraction } & König (1992) \\
\hline & & & & Lantzsch et al. (1996) \\
\hline & 301 & $0.593 \pm 0.012$ & ${ }^{2} \mathrm{H}$ NMR & Leftin et al. (2014) \\
\hline & 301 & $0.604 \pm 0.036$ & ${ }^{13} \mathrm{C}$ NMR & Leftin et al. (2014) \\
\hline & 303 & $0.643 \pm 0.013$ & $\mathrm{X}$-ray scattering & Kučerka et al. (2011) \\
\hline & 303 & $0.683 \pm 0.015$ & $\mathrm{X}$-ray scattering & Kučerka et al. (2005) \\
\hline & 310 & 0.66 & Surface-pressure measur. & Hyslop et al. (1990) \\
\hline & 310 & $0.668 \pm 0.005$ & Small-angle X-ray diffraction & Jerabek et al. (2010) \\
\hline & 321 & $0.662 \pm 0.013$ & ${ }^{2} \mathrm{H}$ NMR & Leftin et al. (2014) \\
\hline & 321 & $0.705 \pm 0.042$ & ${ }^{13} \mathrm{C}$ NMR & Leftin et al. (2014) \\
\hline & 323 & $0.62 \pm 0.01$ & $\mathrm{X}$-ray scattering & Pabst et al. (2000) \\
\hline & 323 & $0.673 \pm 0.013$ & $\mathrm{X}$-ray scattering & Kučerka et al. (2011) \\
\hline 16:0/18:2(n-6) cis PC & 297 & 0.66 & Langmuir film balance & Smaby et al. (1997) \\
\hline 16:0/20:4(n-6) cis PC & 297 & 0.68 & Langmuir film balance & Smaby et al. (1997) \\
\hline 16:0/22:6(n-3)cis PC & 297 & 0.70 & Langmuir film balance & Smaby et al. (1997) \\
\hline \multirow[t]{11}{*}{ 18:0/18:1(n-9)cis PC } & 293 & $0.638 \pm 0.013$ & $\mathrm{X}$-ray scattering & Kučerka et al. (2011) \\
\hline & 303 & $0.614 \pm 0.006$ & ${ }^{2} \mathrm{H}$ NMR and X-ray & Köenig et al. (1997b) \\
\hline & 303 & 0.643 & 'Compressibility' method & Rand and Parsegian (1989) \\
\hline & 303 & $0.655 \pm 0.013$ & $\mathrm{X}$-ray scattering & Kučerka et al. (2011) \\
\hline & 303 & 0.66 & Osmotic pressure technique & Rand et al. (1988) \\
\hline & 303 & 0.66 & Gravimetric method & Rand and Parsegian (1989) \\
\hline & \multirow[t]{2}{*}{303} & \multirow[t]{2}{*}{0.666} & \multirow[t]{2}{*}{${ }^{2} \mathrm{H}$ and ${ }^{31} \mathrm{P}$ NMR } & Separovich and Gawrisch (1996) \\
\hline & & & & Gawrisch and Holte (1996) \\
\hline & 303 & $0.67^{\mathrm{a}}$ & Low-angle X-ray scattering & Pan et al. (2009) \\
\hline & 303 & $0.71^{\mathrm{a}}$ & Wide-angle X-ray scattering & Pan et al. (2009) \\
\hline & 323 & $0.681 \pm 0.014$ & $\mathrm{X}$-ray scattering & Kučerka et al. (2011) \\
\hline \multirow[t]{2}{*}{ 18:0/18:2(n-6)cis $\mathrm{PC}$} & \multirow[t]{2}{*}{303} & \multirow[t]{2}{*}{0.673} & \multirow[t]{2}{*}{${ }^{2} \mathrm{H}$ and ${ }^{31} \mathrm{P} \mathrm{NMR}$} & Separovich and Gawrisch (1996) \\
\hline & & & & Gawrisch and Holte (1996) \\
\hline \multirow[t]{2}{*}{ 18:0/18:3(n-3)cis PC } & \multirow[t]{2}{*}{303} & \multirow[t]{2}{*}{0.666} & \multirow[t]{2}{*}{${ }^{2} \mathrm{H}$ and ${ }^{31} \mathrm{P} \mathrm{NMR}$} & Separovich and Gawrisch (1996) \\
\hline & & & & Gawrisch and Holte (1996) \\
\hline \multirow[t]{2}{*}{ 18:0/20:4(n-6)cis PC } & \multirow[t]{2}{*}{303} & \multirow[t]{2}{*}{0.706} & \multirow[t]{2}{*}{${ }^{2} \mathrm{H}$ and ${ }^{31} \mathrm{P} \mathrm{NMR}$} & Separovich and Gawrisch (1996) \\
\hline & & & & Gawrisch and Holte (1996) \\
\hline \multirow[t]{2}{*}{ 18:0/20:5(n-3)cis PC } & 303 & 0.691 & ${ }^{2} \mathrm{H}$ and ${ }^{31} \mathrm{P}$ NMR & Separovich and Gawrisch (1996) \\
\hline & & & & Gawrisch and Holte (1996) \\
\hline 18:0/22:6(n-3)cis PC & 303 & $0.682 \pm 0.004$ & X-ray diffraction & Eldho et al. (2003) \\
\hline & 303 & $0.692 \pm 0.009$ & ${ }^{2} \mathrm{H}$ NMR and X-ray & Köenig et al. (1997b) \\
\hline & 303 & 0.716 & ${ }^{2} \mathrm{H}$ and ${ }^{31} \mathrm{P}$ NMR & Separovich and Gawrisch (1996) \\
\hline & & & & Gawrisch and Holte (1996) \\
\hline
\end{tabular}


geometrical parameters (bond lengths and bond angles), together with the potentials affecting rotation about bonds, including the effects of steric interactions between atoms and groups which are near neighbors in sequence along the chain; in other words, the short-range effects are determined by interactions between groups separated by only a few bonds. (2) The long-range interactions are dominated by interactions involving pairs of atoms and groups which are remote in the chain sequence, though near to one another in space when involved in mutual interactions; to put it differently, the longrange interactions are determined by interactions between pairs which are separated by many bonds (Flory 1969). work. From the mathematical point of view, to reach this state, the long-range interactions should be excluded.

Thus, the MC simulations were performed here for unperturbed hydrocarbon chains, in which only intramolecular interactions between near neighbours along the chain were included. Let $U$ be the conformational energy of a chain in the unperturbed state, i.e., the short-range interaction energy. The equilibrium properties of all chains were calculated using the classical flexible model (Gö and Scheraga 1976). The average value of the physically observable $\langle H\rangle_{\Theta}$ of an unperturbed molecule in the canonical ensemble for this model is given by

$\langle H\rangle_{\Theta}=\frac{\int_{0}^{2 \pi} \ldots \int_{0}^{2 \pi} H\left(\varphi_{1}, \ldots, \varphi_{N-1}\right) \cdot \exp \left[-U\left(\varphi_{1}, \ldots, \varphi_{N-1}\right) / k_{B} T\right] d \varphi_{1} \ldots d \varphi_{N-1}}{\int_{0}^{2 \pi} \ldots \int_{0}^{2 \pi} \exp \left[-U\left(\varphi_{1}, \ldots, \varphi_{N-1}\right) / k_{B} T\right] d \varphi_{1} \ldots d \varphi_{N-1}}$,

The long-range interactions introduce alterations (perturbation) in the chain configuration obtained when only the short-range interactions are considered. It is important to note that the long-range effect depends not only on the actual volume of the chain group (fragment, unit) but also on its interaction with the solvent; it is reasonable, therefore, to discuss the effective covolume. The covolume for the chain group can be enhanced by use of a 'good' solvent for the chain. It may also be diminished by choice of a 'poor' one barely capable of dissolving the chain. Through judicious selection of solvent and temperature, the finite volume of the group can be compensated exactly by the mutual attractions between chain groups when immersed in the poor solvent (Flory 1969). This state was called the 'Theta $(\Theta)$ point'; where $N$ is the number of carbons of the main chain; $k_{B}$-the Boltzmann constant; $T$-temperature; and $\varphi_{1}, \varphi_{2}, \ldots, \varphi_{N-1}$ are torsion angles of the main chain. Here, in (1) and below in the MC procedure, we keep bond lengths and bond angles equal to their equilibrium values which correspond to the chosen force field parameters; the torsions for all double bonds arranged cis were also fixed at the equilibrium value.

Assume we generate angles $\varphi_{1}, \varphi_{2}, \ldots, \varphi_{N-1}$ with probability density $p\left(\varphi_{1}, \varphi_{2}, \ldots, \varphi_{N-1}\right)$. Then, an assessment $\bar{H}_{\omega}$ of the value $\langle H\rangle_{\Theta}$ by the MC method (Gould et al. 2007; Landau and Binder 2009; Binder and Heermann 2010; Satoh 2011) is

$\bar{H}_{\omega}=\frac{\sum_{v=1}^{\omega} H\left(\varphi_{1}^{v}, \ldots, \varphi_{N-1}^{v}\right) \cdot \exp \left[-U\left(\varphi_{1}^{v}, \ldots, \varphi_{N-1}^{v}\right) / k_{B} T\right] / p\left(\varphi_{1}^{v}, \ldots, \varphi_{N-1}^{v}\right)}{\sum_{v=1}^{\omega} \exp \left[-U\left(\varphi_{1}^{v}, \ldots, \varphi_{N-1}^{v}\right) / k_{B} T\right] / p\left(\varphi_{1}^{v}, \ldots, \varphi_{N-1}^{v}\right)}$.

as this takes place, the perturbation of the chain configuration must vanish and the chain become unperturbed (Flory 1969). Further, according to the prediction by Flory, in the bulk amorphous state, perturbation of the chain configuration must vanish. Neutron scattering experiments were later carried out (Dettenmaier 1978; Yoon and Flory 1978), and the results substantiated this prediction.

The 'unperturbed' state of chain molecules was used as the uniform state of the different hydrocarbon chains in this
Here, $\omega$ is the sample size and $v$ is the number of the current conformation. The value $\bar{H}_{\omega}$ from expression (2) converges to the value $\langle H\rangle_{\Theta}$ from expression (1).

In previous work (Zhurkin and Rabinovich 2015), an important sampling technique was developed for the efficient generation of chain conformations, with continuous variation of all single $\mathrm{C}-\mathrm{C}$ bond torsions within $[0,2 \pi]$ range. The conformations were generated using the probability density

$$
p\left(\varphi_{1}, \ldots, \varphi_{N-1}\right) \approx \frac{\exp \left[-U\left(\varphi_{1}, \ldots, \varphi_{N-1}\right) / k_{B} T\right]}{\int_{0}^{2 \pi} \ldots \int_{0}^{2 \pi} \exp \left[-U\left(\varphi_{1}, \ldots, \varphi_{N-1}\right) / k_{B} T\right] d \varphi_{1} \ldots d \varphi_{N-1}} .
$$


To calculate the conformational energy $U$ of a hydrocarbon chain in the unperturbed state (the short-range interactions energy), a scheme of interdependence of each of three torsions along the chain was taken into account in our work. The energy $U$ was calculated as the sum of energies $U_{m}\left(\varphi_{\gamma}, \varphi_{\gamma+1}, \varphi_{\gamma+2}\right)$ of $N_{f}$ structural units (e.g., $N_{f}=N-3$ for a saturated chain with $N$ carbons):

$U=\sum_{\gamma=1}^{N_{f}} U_{m_{\gamma}}\left(\varphi_{\gamma}, \varphi_{\gamma+1}, \varphi_{\gamma+2}\right)$.

where $\varphi_{\gamma}, \varphi_{\gamma+1}, \varphi_{\gamma+2}$ are torsion angles, and $m$ is the structural unit type. The units reproduced precisely the structure of various chain fragments.

The energy $U$ is arranged [according to (4)] in such a way that it is possible to calculate energy $U_{m_{\gamma}}$ of any $m$ unit at the preliminary step, before MC simulation of the chain. To calculate the energies of all units and chains as a whole, the same force field parameters as in the MD simulations were used (CHARMM27; Feller and MacKerell, Jr. 2000) with modifications performed in the paper (Högberg et al. 2008).

To construct a chain of the above-mentioned structure and then calculate the energy $U$ according to expression (4), several of the 16 structural units presented in Fig. 4 should be properly combined. Three variable torsions in each unit in Fig. 4 are marked by red arrows. It is seen from expression (4) that the structural units in a chain should be connected in such a way that each two consecutive (neighboring) units have two mutually variable torsions.

When calculating $U_{m}$ of each structural unit, the torsion energy, non-bonded energy and electrostatic energy from the force field were considered; bond lengths and bond angles were fixed at equilibrium values. Therefore
$U$ according to (4) only approximately; nevertheless, it is quite sufficient for the next step of the algorithm. An exact calculation of the energy $U$ of each conformation is carried out after it has been generated-see below: it will be denoted as $U_{\text {units }}$ in the final assessment $\bar{H}_{\omega}$ of the value $\langle H\rangle_{\Theta}$.

It is possible to demonstrate here an 'interdependence phenomenon' of three consecutive variable torsions along the chain by the example of structural unit 9 from Fig. 4 . Energy $U_{9}\left(\varphi_{1}, \varphi_{2}, \varphi_{3}\right)$ of unit 9 was calculated (tabulated) and presented in Fig. 5 in the form of six two-dimensional energy maps containing equienergy contours, i.e., lines connecting the points of equal energies.

The variants presented in Fig. 5 contain

1. Equienergy contours for $\left(\varphi_{1}, \varphi_{2}\right)$ pair at two fixed values of $\varphi_{3}\left(\varphi_{3}=60^{\circ}\right.$, Fig. 5a, and $\varphi_{3}=120^{\circ}$, Fig. 5b),

2. Equienergy contours for $\left(\varphi_{1}, \varphi_{3}\right)$ pair at two fixed values of $\varphi_{2}\left(\varphi_{2}=60^{\circ}\right.$, Fig. $5 \mathrm{c}$, and $\varphi_{2}=120^{\circ}$, Fig. 5d),

3. Equienergy contours for $\left(\varphi_{2}, \varphi_{3}\right)$ pair at two fixed values of $\varphi_{1}\left(\varphi_{1}=60^{\circ}\right.$, Fig. 5e, and $\varphi_{1}=120^{\circ}$, Fig. 5f $)$.

Equienergy contours for a pair of torsions can, of course, be demonstrated at any fixed value of the third torsion angle (not only at $60^{\circ}$ and $120^{\circ}$ ). It is seen that map (a) of Fig. 5 is significantly different from map (b) of this figure; a large difference is observed for maps (c) and (d), maps (e) and (f), i.e., dependence of energy $U_{9}$ on torsion angles $\varphi_{1}, \varphi_{2}, \varphi_{3}$ is pronounced (in other words, interdependence exists between the three torsions).

To generate the density (3) for a given chain, a special technique was developed. Let $N_{f}=N-3$ for simplicity; then, we can substitute $U$ from (3) by expression (4) and rewrite expression (3) in a different form:

$$
p\left(\varphi_{1}, \ldots, \varphi_{N-1}\right) \approx \frac{\prod_{\gamma=1}^{N-3} \exp \left[-U_{m_{\gamma}}\left(\varphi_{\gamma}, \varphi_{\gamma+1}, \varphi_{\gamma+2}\right) / k_{B} T\right]}{\int_{0}^{2 \pi} \ldots \int_{0}^{2 \pi N-3} \prod_{\gamma=1} \exp \left[-U_{m_{\gamma}}\left(\varphi_{\gamma}, \varphi_{\gamma+1}, \varphi_{\gamma+2}\right) / k_{B} T\right] \cdot d \varphi_{1} \ldots d \varphi_{N-1}} .
$$

bond length energy and bond angle energy were constant. In the calculations, multipliers of $1 / 2$ and $1 / 3$ were used for some energy items to exclude the possibility of double (or triple) summation of any energy items in the final expression (4). The multiplier $1 / 2$ was used for the energy items which are strictly dependent on two variable torsions, and 1/3 was used for items strictly dependent on one variable torsion. The energy items that are strictly dependent on the three variable torsions of the considered structural unit were accounted without multipliers (i.e., a multiplier was equal to 1). It should be recorded that, at this step, it is possible to calculate the short-range energy
The energy $U_{m}\left(\varphi_{\gamma}, \varphi_{\gamma+1}, \varphi_{\gamma+2}\right)$ of each structural unit $m$ was tabulated with a step of $1^{\circ}$. Then $\exp \left[-U_{m}\left(\varphi_{\gamma}, \varphi_{\gamma+1}, \varphi_{\gamma+2}\right) / k_{B} T\right]$ values under given $T$ were calculated, and integrals

$$
\int_{0}^{2 \pi} \int_{0}^{2 \pi} \int_{0}^{2 \pi} \exp \left[-U_{m}\left(\varphi_{\gamma}, \varphi_{\gamma+1}, \varphi_{\gamma+2}\right) / k_{B} T\right] d \varphi_{\gamma} d \varphi_{\gamma+1} d \varphi_{\gamma+2}
$$

were computed numerically. Then, the configurational space of torsion angles $\varphi_{\gamma}, \varphi_{\gamma+1}, \varphi_{\gamma+2}$ of each chain's unit $m$, where $0 \leq \varphi_{\gamma} \leq 2 \pi, 0 \leq \varphi_{\gamma+1} \leq 2 \pi, 0 \leq \varphi_{\gamma+2} \leq 2 \pi$ (i.e., a 'cube'), was divided numerically into $100^{3}=$ 


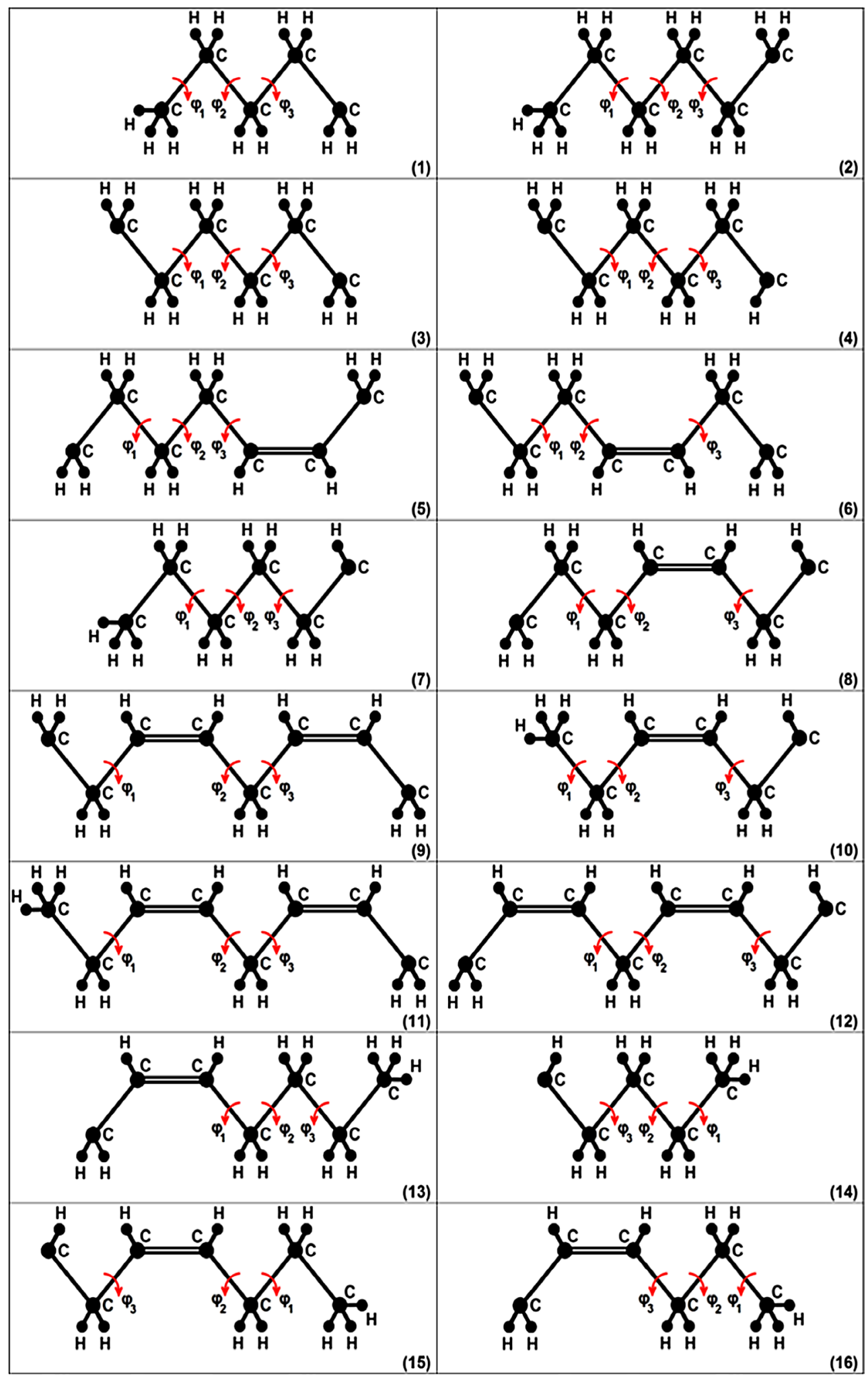

Fig. 4 Sixteen structural units; to construct a linear hydrocarbon (n-alkane or n-alkene) chain of the structure, e.g., as in Fig. 2, that is typical for the biomembrane phospholipid chain structure (Fig. 1), and calculate the energy $U$ according to expression (4), several of the presented units should be properly combined. Three variable torsions in each unit are marked by red arrows. The number at the bottom right of the unit is the unit's number 

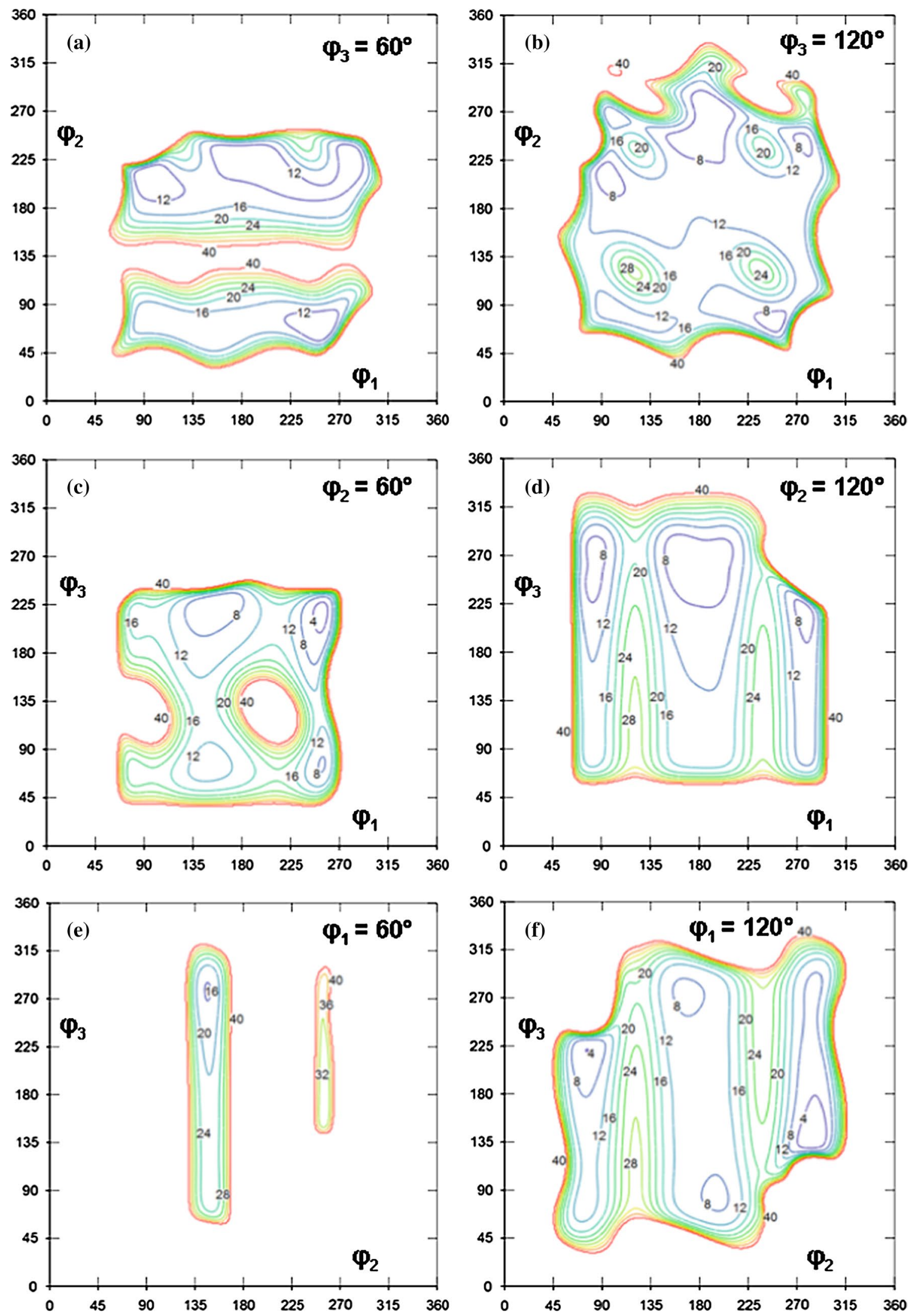

Fig. 5 Six two-dimensional energy maps of structural unit 9 from Fig. 4. The value $0^{\circ}$ of any torsions (angles $\varphi_{1}, \varphi_{2}$ and $\varphi_{3}$ ) corresponds to the eclipsed conformation. The numbers near equienergetic

contours are energies $(\mathrm{kJ} / \mathrm{mol})$. The energy of each map is measured from the global energy minimum of structural unit 9 
$1,000,000$ parallelepipeds ('states') in such a way that they all have equal Boltzmann realization probabilities under given $T$. As a result, boundaries between the 'states' along three directions (torsions) in angle units were calculated; to do that, a special mathematical algorithm was developed. The idea of the algorithm is as follows. At first, values of boundaries along $\varphi_{\gamma}$ axis were calculated using a recurrent relationship, to divide the 'cube' onto 100 quadratic $(2 \pi \times 2 \pi)$ strata ('layers') having equal realization probabilities and, as a consequence, different widths. Then, each stratum, using a recurrent relationship, was numerically divided along the second axis, $\varphi_{\gamma+1}$, onto 100 'columns' ('rods') in such a way that all 'columns' have equal realization probabilities (and, because of this, different sizes); the values of boundaries between the 'columns' were obtained. Finally, each 'column' was similarly divided along the third axis, $\varphi_{\gamma+2}$, onto 100 equiprobable parallelepipeds ('states') and, hence, all sizes (edge lengths in angle units) of the parallelepipeds are not equal to each other. It is evident that with this method of splitting, the boundaries of parallelepipeds of each structural unit $m$ gather in the areas where energy minima, i.e. the number of states (parallelepipeds) in the energy minima is much more than those around the maxima.

The calculated boundaries between 1,000,000 equiprobable parallelepipeds for each chain's unit $m$ are then used in MC simulations of the different hydrocarbon chains. The current chain conformation (a set of values of torsions along the chain) is generated randomly by selection of the stratum, column and parallelepiped numbers and then by selection of exact values of the torsions inside the chosen parallelepipeds. In doing so, the proper sequences of structural units of the given chain and the torsion numbers along the chain are obeyed.

The final assessment $\bar{H}_{\omega}$ of the value $\langle H\rangle_{\Theta}$ within the methodology can be obtained by

$\bar{H}_{\omega}=\frac{\sum_{v=1}^{\omega} H\left(\varphi_{1}^{\nu}, \ldots, \varphi_{N-1}^{v}\right) \cdot \exp \left[-U_{\text {units }}\left(\varphi_{1}^{v}, \ldots, \varphi_{N-1}^{v}\right) / k_{B} T\right] \cdot W^{v}}{\sum_{\nu=1}^{\omega} \exp \left[-U_{\text {units }}\left(\varphi_{1}^{v}, \ldots, \varphi_{N-1}^{v}\right) / k_{B} T\right] \cdot W^{v}}$.

where

$W^{\nu}=\left[\left(L_{1}\right)_{m_{1}, \lambda_{1,1}^{v}}\left(L_{2}\right)_{m_{1}, \lambda_{2,1}^{v}}\left(L_{3}\right)_{m_{1}, \lambda_{3,1}^{v}}\right] \cdot \prod_{\gamma=2}^{N-3}\left(L_{3}\right)_{m_{\gamma}, \lambda_{3, \gamma}^{v}}$

Here, $\left(L_{1}\right)_{m_{\gamma}, \lambda_{1, \gamma}^{\nu}},\left(L_{2}\right)_{m_{\gamma}, \lambda_{2, \gamma}^{v}}$ and $\left(L_{3}\right)_{m_{\gamma}, \lambda_{3, \gamma}^{v}}$ are sizes (edge lengths in angle units) of a parallelepiped randomly chosen for the $v$-th conformation of the chain in the molecular unit number $\gamma$ of type $m ; \lambda_{1, \gamma}^{v}, \lambda_{2, \gamma}^{v}$ and $\lambda_{3, \gamma}^{v}$ are random numbers of the three edges of the chosen parallelepiped;
$U_{\text {units }}\left(\varphi_{1}^{v}, \ldots, \varphi_{N-1}^{v}\right)$ is the short-range interactions energy of the generated $v$-th chain conformation, properly calculated using all terms of the force field: all bond length and angle energies, all torsion (dihedral) angle energies, out-ofplane energies, Urey-Bradley terms, and non-bonded and electrostatic interactions energies for such pairs of atoms which are included into the sequence of structural units of the given chain. Since all values of the torsions in the generated conformation are already known, the short-range energy $U_{\text {units }}$ is calculated correctly [in contrast to the energy $U$ calculated approximately from expression (4)].

Thus, in the assessment (6), the probability of generation of each chain conformation and probability of its realization are calculated, and hence we obtain $\bar{H}_{\omega} \stackrel{\omega \rightarrow \infty}{\longrightarrow}\langle H\rangle_{\Theta}$. To calculate average characteristics, approximately $10^{12}$ conformations of each chain were generated in the present work.

\section{Results and discussion}

Average distances between terminal carbon atoms of the chains (end-to-end distances) considered in the unperturbed state, $\langle h\rangle_{\Theta}$, and those of PC lipid chains in liquid crystalline bilayers, $\langle h\rangle_{b i}$, were calculated. The obtained data are presented in Table 8 .

Table 8 shows that $\langle h\rangle_{\Theta}$ values are somewhat less than $\langle h\rangle_{b i l}$. This is because only one hydrocarbon chain terminus in the lipid molecule is free in space; the other one is chemically linked to the head group. Due to interactions of all lipid molecules with their neighbors and water molecules the lipids' head groups are arranged in the vicinity to each other. Therefore, possibilities of rotations around several $\mathrm{C}-\mathrm{C}$ bonds adjoining the head groups are more restricted than those for the $\mathrm{C}-\mathrm{C}$ bonds of the opposite end of the chain, so the chain region near the head groups is more stretched, in contrast to the unperturbed chain in which both ends are free.

To compare the results quantitatively, the relative (in percentage) difference $\delta$ between values $\langle h\rangle_{\text {bil }}$ and $\langle h\rangle_{\Theta}$ for each chain was calculated:

$\delta=\left[\left(\langle h\rangle_{\text {bil }}-\langle h\rangle_{\Theta}\right) /\langle h\rangle_{\text {bil }}\right] \cdot 100$.

The values of $\delta$ are also presented in Table 8. The calculations showed that the relative difference $\delta$ between both states increases as the number of carbons and/or number of double bonds in the chain increase; $\delta$ is approximately equal to $8-10 \%$ (or $\sim 9 \%$ ) for saturated $16: 0$ and 18:0 chains; to $10-15 \%$ for unsaturated chains with $N=18$, $d=1-5$; to $16-17 \%$ for PU chains with $N=20, d=4-5$ and maximum $\sim 19 \%$ for $22: 6(n-3)$ cis chain.

In this connection, a remark should be made. It is possible to divide interactions in the bilayers into three parts: 
Table 8 Average end-to-end distances, $\langle h\rangle_{\Theta}$ for unperturbed hydrocarbon chains and $\langle h\rangle_{b i l}$ for the acyl chains in liquid crystalline phosphatidylcholine (PC) bilayers obtained by computer simulations of the present work; $T=303 \mathrm{~K}$

\begin{tabular}{llllll}
\hline $\begin{array}{l}\text { Hydrocarbon chain, } \\
\text { MC simulation }\end{array}$ & $\begin{array}{l}\langle h\rangle_{\Theta}\left( \pm \Delta_{1}\right)^{\mathrm{a}},[\mathrm{nm}] \\
\text { Unperturbed state } \\
\text { MC simulation }\end{array}$ & $\begin{array}{l}\text { Acyl chain, } \\
\text { MD simulation }\end{array}$ & $\begin{array}{l}\langle h\rangle_{\text {bil }}\left( \pm \Delta_{2}\right)^{\mathrm{b}},[\mathrm{nm}] \\
16: 0 / \ldots \text { PC bilayer } \\
\text { MD simulation }\end{array}$ & $\begin{array}{l}\langle h\rangle_{\text {bil }}\left( \pm \Delta_{2}\right)^{\mathrm{b}},[\mathrm{nm}] \\
18: 0 / \ldots \text { PC bilayer } \\
\text { MD simulation }\end{array}$ & $\begin{array}{l}\text { Relat. diff. expression } \\
(8)) \\
\delta, \%\end{array}$ \\
\hline alk-16:0 & $1.395( \pm 0.001)$ & $16: 0$ & $1.503-1.520^{\mathrm{c}}( \pm 0.001)$ & & $7.2-8.2^{\mathrm{e}}$ \\
alk-18:0 & $1.528( \pm 0.001)$ & $18: 0$ & & $1.665-1.699^{\mathrm{d}}( \pm 0.001)$ & $8.3-10.1^{\mathrm{f}}$ \\
alk-18:1 & $1.374( \pm 0.001)$ & $18: 1$ & $1.532( \pm 0.001)$ & $1.531( \pm 0.001)$ & $10.3 ; 10.3^{\mathrm{g}}$ \\
alk-18:2 & $1.293( \pm 0.001)$ & $18: 2$ & $1.478( \pm 0.001)$ & $1.491( \pm 0.001)$ & $12.5 ; 13.3^{\mathrm{g}}$ \\
alk-18:3 & $1.261( \pm 0.001)$ & $18: 3$ & $1.447( \pm 0.001)$ & $1.446( \pm 0.001)$ & $12.8 ; 12.8^{\mathrm{g}}$ \\
alk-18:4 & $1.194( \pm 0.003)$ & $18: 4$ & $1.398( \pm 0.001)$ & $1.402( \pm 0.001)$ & $14.6 ; 14.8^{\mathrm{g}}$ \\
alk-18:5 & $1.165( \pm 0.008)$ & $18: 5$ & $1.372( \pm 0.001)$ & $1.373( \pm 0.001)$ & $15.1 ; 15.1^{\mathrm{g}}$ \\
alk-20:4 & $1.290( \pm 0.002)$ & $20: 4$ & $1.544( \pm 0.001)$ & $1.538( \pm 0.001)$ & $16.5 ; 16.1^{\mathrm{g}}$ \\
alk-20:5 & $1.254( \pm 0.001)$ & $20: 5$ & $1.497( \pm 0.001)$ & $1.509( \pm 0.001)$ & $16.2 ; 16.9^{\mathrm{g}}$ \\
alk-22:6 & $1.328( \pm 0.005)$ & $22: 6$ & $1.624( \pm 0.001)$ & $1.645( \pm 0.001)$ & $18.2 ; 19.3^{\mathrm{g}}$ \\
\hline
\end{tabular}

${ }^{\text {a }}$ stat. error $\Delta_{1}$ evaluated from the variance of $\sim 10^{12}$ conformations

${ }^{\mathrm{b}}$ stat. error $\Delta_{2}$ for 20-100 ns evaluated from the variance of $10 \mathrm{~ns}$ block averages

${ }^{\mathrm{c}}$ range of $\langle h\rangle_{\text {bil }}$ for 16:0 acyl chain in eight mixed-chain 16:0/... PC bilayers

${ }^{\mathrm{d}}$ range of $\langle h\rangle_{\mathrm{bil}}$ for 18:0 acyl chain in eight mixed-chain 18:0/... PC bilayers

${ }^{\mathrm{e}}$ range of $\delta$ values for 16:0 acyl chain in eight mixed-chain 16:0/... PC bilayers

${ }^{\mathrm{f}}$ range of $\delta$ values for 18:0 acyl chain in eight mixed-chain 18:0/... PC bilayers

$\mathrm{g}$ values of $\delta$ for this unsaturated acyl chain in 16:0/... PC and 18:0/... PC bilayers, respectively

intramolecular short-range, intramolecular long-range interactions (Flory 1969) of the chains, and intermolecular interactions between the chains and the neighboring chains (and PC head groups). Unperturbed hydrocarbon chain properties are fully defined by the short-range interaction energy (Flory 1969). Therefore, the value of $\delta$ can be considered as an assessment of the influence of the longrange interactions inside the chain and interactions with the neighboring chains and PC head groups of the lipid bilayer on the distance $\langle h\rangle_{\text {bil }}$, compared with the influence of only short-range interactions inside the chain on the $\langle h\rangle_{\Theta}$.

While conformations of the unperturbed chains are not the same as those in liquid crystalline bilayers, the relative difference $\delta$ between the end-to-end distances $\langle h\rangle_{b i l}$ and $\langle h\rangle_{\Theta}$ of the considered typical acyls was found to be comparatively moderate; it is approximately equal to $9-19 \%$.

Therefore, such properties of the listed FA chains of phospholipids in bilayers, as $\langle h\rangle_{\text {bil }}$ are significantly determined by the short-range interactions in the chains (indeed, it is determined approximately by $81-91 \%$ ). There is good reason to believe that other geometrical properties of these chains are also determined mainly by their short-range interactions. We mention that the considered saturated and unsaturated chains are widespread, typical constituents of phospholipids, so the obtained result seems to be valuable for biomembranes. In other words, the $\delta$ value seems to be of the same order of magnitude for most of different membrane hydrocarbon chains with methylene-interrupted cis double bonds.

It should be also pointed out, that a relationship between $\langle h\rangle_{\Theta}$ and the number $d$ of double bonds for the unperturbed hydrocarbon chains with constant number $N$ of carbon atoms is the same as $\langle h\rangle_{\text {bil }}$ for acyl chains of PC molecules in bilayers: both values decrease as $d$ increases (Table 8). In other words, the trends in changes of $\langle h\rangle_{\Theta}$ and $\langle h\rangle_{\text {bil }}$ in the order 18:0 $\rightarrow 18: 1 \rightarrow 18: 2 \rightarrow 18: 3 \rightarrow 18: 4 \rightarrow 18: 5$ are the same; the trends in the order 20:4 $\rightarrow$ 20:5 are also the same.

This decrease is obviously caused mainly by extension of the chain segment with double bonds, in which energy minima are wide and correspond to various collapsed chain conformations, and by shortening of chain's saturated segments in which the main energy minimum corresponds to the extended chain conformations (Flory 1969).

It is possible to consider also the trends of both discussed values of $\langle h\rangle$ in the order 18:4 $\rightarrow$ 20:4 and the trends in the order 18:5 $\rightarrow 20: 5$, i.e., relationships between $\langle h\rangle_{\Theta}$, $\langle h\rangle_{\text {bil }}$ and number $N$ of carbon atoms under constant number $d$ of double bonds. Table 8 shows that they are similar to each other, respectively. The discussed trends are shown schematically in Fig. 6. Of course, more rigorous treatment requires consideration also of the value of the parameter $j$ in all trends, i.e., the third structure parameter of the chain in the expression $N: d(n-j)$ cis that means the positions of double bonds along the chain. In particular, $j$ differs for the 


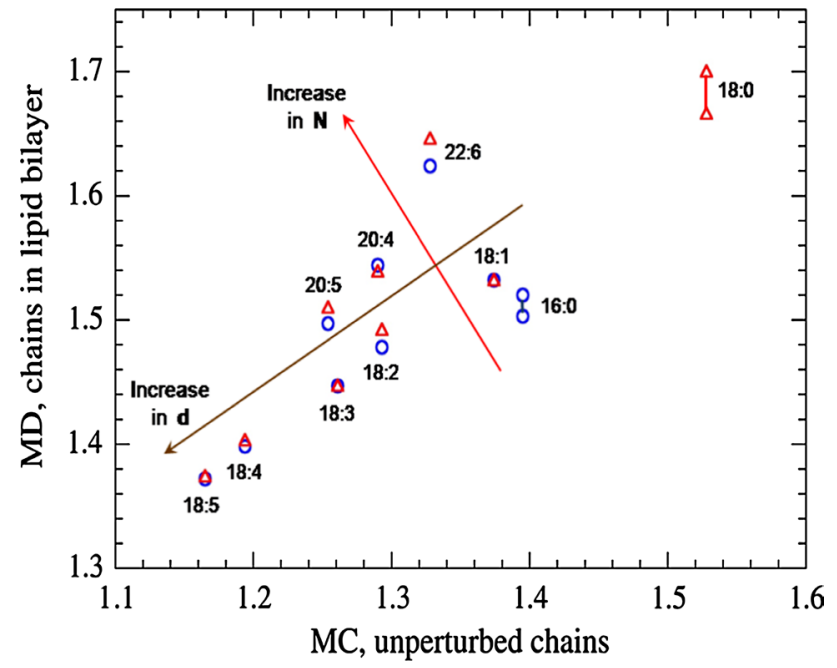

Fig. 6 Average end-to-end distances $\langle h\rangle_{\Theta}$, [nm], for unperturbed hydrocarbon chains obtained by Monte Carlo (MC) simulations, and $\langle h\rangle_{\text {bil }},[\mathrm{nm}]$, for the same acyl chains in liquid crystalline phosphatidylcholine (PC) bilayers obtained by molecular dynamics (MD) simulations (triangles for the marked chains in 18:0/.. PC bilayers, circles for the marked chains in 16:0/... PC bilayers). The ranges for saturated acyl chains 16:0 and 18:0 are obtained for eight appropriate mixed-chain PC bilayers. Computer simulations of the present work, $T=303 \mathrm{~K}$. Arrows show qualitatively trends in $\langle h\rangle_{\Theta}$ and $\langle h\rangle_{\text {bil }}$. To compare the obtained trends, the same names used here both for acyls (ordinate axis) and hydrocarbon chains (abscissa axis)

considered chains 18:4 and 20:4, 20:4 and 20:5, and for some chains with $N=18$.

To our knowledge, a quantitative assessment of the difference between properties of the two chain states (in particular, the difference in $\langle h\rangle$ values) was obtained for the first time in the present work, while a qualitative similarity of chain properties in the two states was discussed in the literature before. For instance, it has been concluded (Rabinovich et al. 2003) that the bond-order parameters and orientation distribution characteristics of the chains in the lipid monolayer and bilayer 'liquid' regions, as found in experiments and in MD computer simulation models (Rabinovich et al. 1999a, b, 2000; Rabinovich and Balabaev 2001), are qualitatively similar to the intramolecular order parameters and the intramolecular bond orientation distributions in single unperturbed (Flory 1969) unsaturated hydrocarbon chains previously investigated with MC simulations (Rabinovich and Ripatti 1999, 2000). Therefore, the behavior of the acyl chains in the liquid region of lipid bilayers (somewhat remote from the membrane-water interface) is dominated by the intramolecular short-range interactions. The long-range interactions of the segments of the lipids in this region of the bilayer and the interactions with the bilayer-water interface may be considered as a disturbance: the intermolecular interactions are largely used to orient the lipid molecules in the direction of the membrane normal.

Thus, from the two above-mentioned facts (about a comparatively moderate quantitative difference in the $\langle h\rangle_{\text {bil }}$ and $\langle h\rangle_{\Theta}$ values of the chains and the similarity of their trends), a common conclusion can be made: to treat and analyse a number of processes in biological membranes (e.g., changes in FA composition caused by the environmental changes such as temperature and pressure), it is possible to use, at least as the first approximation, the relationships between structure and properties obtained for the unperturbed hydrocarbon chains. This seems not unreasonable: biomembranes are known to contain a wide variety of FA chains; the available 'bilayer' relations between their structure and property are incomplete and insufficient for the analysis, whereas the properties of the unperturbed chains and corresponding 'structure-property' relationships have been already studied for tens of variants (see, e.g., Zhurkin and Rabinovich 2015).

\section{Conclusions}

The average characteristics of hydrocarbon chains calculated in the unperturbed state (which is fully defined by short-range interaction energies) make it possible to estimate the influence of additional energy components on the state of these chains, if they are under other conditions or located in other systems. For the same temperature and force field parameters, it is acceptable to use any characteristic as a criterion. The average end-to-end distance of the chains was chosen as such a criterion in the computer simulations carried out in this work. The relationships between structure and the average end-to-end distances obtained for the considered unperturbed chains were shown to be qualitatively similar to those of lipid chains in bilayers. Such data for the majority of possible lipid acyls in bilayers (as a rule, it is several tens of chains or more) are yet unknown because MD simulations of various lipid bilayers are very time-consuming. On this basis, it is reasonable to investigate the unperturbed hydrocarbon chains instead. As a first approximation of the desired 'structure-property' relationships for the lipid chains in bilayers, the corresponding relationships for the unperturbed chains can be used.

Acknowledgements This work has been supported by the federal budget assignment no. 0221-2014-0033 of the Russian Federation (to $\mathrm{AR}$ and DZ), the Swedish Research Council (Vetenskapsrådet, grant 621-2013-4260, to AL), and EU FP7 MembraneNanoPart project (to $\mathrm{AR}, \mathrm{AL}$ and DZ). The authors thank the Swedish National Infrastructure for Computing (SNIC) for granting computer facilities.

Open Access This article is distributed under the terms of the Creative Commons Attribution 4.0 International License (http:// 
creativecommons.org/licenses/by/4.0/), which permits unrestricted use, distribution, and reproduction in any medium, provided you give appropriate credit to the original author(s) and the source, provide a link to the Creative Commons license, and indicate if changes were made.

\section{References}

Allen MP, Tildesley DJ (1987) Computer simulations of liquids, 2nd edn. Clarendon, Oxford

Baoukina S, Tieleman DP (2016) Computer simulations of lung surfactant. Biochim Biophys Acta 1858(10):2431-2440. doi:10.1016/j.bbamem.2016.02.030

Barry JA, Trouard TP, Salmon A, Brown MF (1991) Low-temperature ${ }^{2} \mathrm{H}$ NMR spectroscopy of phospholipid bilayers containing docosahexaenoyl (22:6 $\omega 3$ ) chains. Biochemistry 30(34):8386-8394

Bennett WFD, Tieleman DP (2013) Computer simulations of lipid membrane domains. Biochim Biophys Acta 1828(8):17651776. doi:10.1016/j.bbamem.2013.03.004

Benz RW, Castro-Román F, Tobias DJ, White SH (2005) Experimental validation of molecular dynamics simulations of lipid bilayers: a new approach. Biophys J 88(2):805-817

Berendsen HJC (2007) Simulating the physical world: hierarchical modeling from quantum mechanics to fluid dynamics. Cambridge University Press, Cambridge

Bhaswant M, Poudyal H, Brown L (2015) Mechanisms of enhanced insulin secretion and sensitivity with n-3 unsaturated fatty acids. J Nutr Biochem 26:571-584. doi:10.1016/j.jnutbio.2015.02.001

Binder K, Heermann DW (2010) Monte Carlo simulations in statistical physics. An introduction. Springer, Heidelberg

Boggs JM, Tümmler B (1993) Interdigitated gel phase bilayers formed by unsaturated synthetic and bacterial glycerolipids in the presence of polymyxin B and glycerol. Biochim Biophys Acta 1145(1):42-50

Broniec A, Goto M, Matsuki H (2009) A peculiar phase transition of plasmalogen bilayer membrane under high pressure. Langmuir 25(19): 11,265-11,268

Brooks S, Gelman A, Jones GL, Meng X-L (2011) Handbook of Markov Chain Monte Carlo. Chapman and Hall/CRC, London

Bryant G, Pope JM, Wolfe J (1992) Low hydration phase properties of phospholipid mixtures. Eur Biophys J 21(3):223-232

Bunker A, Magarkar A (2016) Rational design of liposomal drug delivery systems, a review: combined experimental and computational studies of lipid membranes, liposomes and their pegylation. Biochim Biophys Acta 1858(10):2334-2352. doi:10.1016/j.bbamem.2016.02.025

Carrin ME, Carelli AA (2010) Peanut oil: compositional data. Eur J Lipid Sci Technol 112:697-707

Cook HW, McMaster CR (2002) Fatty acid desaturation and chain elongation in eukaryotes. In: Vance DE, Vance JE (eds) New comprehensive biochemistry Biochemistry of Lipids, Lipoproteins and Membranes, vol 36, 4th edn. Elsevier, New York, pp 181-204

Coolbear KP, Berde CB, Keough KMW (1983) Gel to liquid-crystalline phase transitions of aqueous dispersions of polyunsaturated mixed-acid phosphatidylcholines. Biochemistry 22(6):1466-1473

Coolbear KP, Keough KMW (1983) Lipid oxidation and gel to liquid-crystalline transition temperatures of synthetic polyunsaturated mixed-acid phosphatidylcholines. Biochim Biophys Acta 732:531-540

Cunningham BA, Tsujita T, Brockman H (1989) Enzymatic and physical characterization of diacylglycerol-phosphatidylcholine interactions in bilayers and monolayers. Biochemistry 28(1):32-40

Curatolo W (1985) The effects of ethylene glycol and dimethyl sulfoxide on cerebroside metastability. Biochim Biophys Acta 817(1):134-138

Curatolo W, Sears B, Neuringer L (1985) A calorimetry and deuterium NMR study of mixed model membranes of 1-palmitoyl2-oleylphosphatidylcholine and saturated phosphatidylcholines. Biochim Biophys Acta 817(2):261-270

Curatolo W (1986) The interactions of 1-palmitoyl-2-oleylphosphatidylcholine and bovine brain cerebroside. Biochim Biophys Acta 861:373-376

Dai MC, Chiche HB, Düzgünes N, Ayanoglu E, Djerassi C (1991) Phospholipid studies of marine organisms: 26. interactions of some marine sterols with 1-stearoyl-2-oleoyl phosphatidylcholine (SOPC) in model membranes. Chem Phys Lipids 59(3):245-253

Davis PJ, Coolbear KP, Keough KMW (1980) Differential scanning calorimetric studies of the thermotropic phase behaviour of membranes composed of dipalmitoyllecithin and mixed-acid unsaturated lecithins. Can J Biochem 58(10):851-858

Davis PJ, Fleming BD, Coolbear KP, Keough KMW (1981) Gel to liquid-crystalline transition temperatures of water dispersions of two pairs of positional isomers of unsaturated mixed-acid. Biochemistry 20(12):3633-3636

Davis PJ, Kariel N, Keough KMW (1986) Gel to liquid-crystalline transitions of aqueous dispersions of positional isomers of a heteroacid unsaturated phosphatidylcholine mixed with epicholesterol and cholesterol. Biochim Biophys Acta 856(2):395-398

Davis PJ, Keough KMW (1983) Differential scanning calorimetric studies of aqueous dispersions of mixtures of cholesterol with some mixed-acid and single-acid phosphatidylcholines. Biochemistry 22(26):6334-6340

Deese AJ, Dratz EA, Dahlquist FW, Paddy MR (1981) Interaction of rhodopsin with two unsaturated phosphatidylcholines: a deuterium nuclear magnetic resonance study. Biochemistry 20(22):6420-6427

Dekker CJ, van Kessel WSMG, Klomp JPG, Pieters J, de Kruijff B (1983) Synthesis and polymorphic phase behaviour of polyunsaturated phosphatidylcholines and phosphatidylethanolamines. Chem Phys Lipids 33(1):93-106

D'Eliseo D, Velotti F (2016) Omega-3 fatty acids and cancer cell cytotoxicity: implications for multi-targeted cancer therapy. $\mathbf{J}$ Clin Med 5(2):15. doi:10.3390/jcm5020015

Dettenmaier M (1978) Conformation of n-alkane molecules in the melt and in cyclohexane solution studied by small-angle neutron scattering. J Chem Phys 68(5):2319-2322

Dirand M, Bouroukba M, Briard AJ, Chevallier V, Petitjean D, Corriou JP (2002) Temperatures and enthalpies of (solid + solid) and (solid + liquid) transitions of n-alkanes. J Chem Thermodyn 34:1255-1277. doi:10.1006/jcht.2002.0978

Diwakar BT, Dutta PK, Lokesh BR, Naidu KA (2010) Physicochemical properties of garden cress (Lepidium sativum L.) seed oil. J Am Oil Chem Soc 87:539-548

(2009) Docosahexaenoic acid (DHA). Altern Med Rev 14(4):391-399

Dumaual AC, Jenski LJ, Stillwell W (2000) Liquid crystalline/gel state phase separation in docosahexaenoic acid-containing bilayers and monolayers. Biochim Biophys Acta 1463:395-406

Eldho NV, Feller SE, Tristram-Nagle S, Polozov IV, Gawrisch K (2003) Polyunsaturated docosahexaenoic vs docosapentaenoic acid differences in lipid matrix properties from the loss of one double bond. J Am Chem Soc 125:6409-6421

Feller SE, Gawrisch K, MacKerell AD (2002) Polyunsaturated fatty acids in lipid bilayers: intrinsic and environmental contributions to their unique physical properties. J Am Chem Soc $124: 318-326$ 
Feller SE, MacKerell AD Jr (2000) An improved empirical potential energy function for molecular simulations of phospholipids. J Phys Chem B 104:7510-7515

Fincham D (1994) Optimization of the Ewald sum for large systems. Mol Simul 13:1-9

Flory PJ (1969) Statistical mechanics of chain molecules. Interscience, New York

Gawrisch K, Holte LL (1996) NMR investigations of non-lamellar phase promoters in the lamellar phase state. Chem Phys Lipids 81:105-116

Ghosh R (1988) ${ }^{31} \mathrm{P}$ and ${ }^{2} \mathrm{H}$ NMR studies of structure and motion in bilayers of phosphatidylcholine and phosphatidylethanolamine. Biochemistry 27(20):7750-7758

Gö N, Scheraga HA (1976) On the use of classical statistical mechanics in the treatment of polymer chain conformations. Macromolecules 9(4):535-542

Gould H, Tobochnik J, Christian W (2007) An introduction to computer simulation methods: applications to physical systems. Pearson, San Francisco

Guil-Guerrero JL (2007) Stearidonic acid (18:4n-3): metabolism, nutritional importance, medical uses and natural sources. Eur J Lipid Sci Technol 109:1226-1236

Hernandez-Borrell J, Keough KMW (1993) Heteroacid phosphatidylcholines with different amounts of unsaturation respond differently to cholesterol. Biochim Biophys Acta 1153:277-282

Högberg CJ, Nikitin AM, Lyubartsev AP (2008) Modification of the CHARMM force field for DMPC lipid bilayer. J Comp Chem 29:2359-2369

Holte LL, Peter SA, Sinnwell TM, Gawrisch K (1995) ${ }^{2} \mathrm{H}$ nuclear magnetic resonance order parameter profiles suggest a change of molecular shape for phosphatidylcholines containing a polyunsaturated acyl chain. Biophys J 68(6):2396-2403

Huang C, Li S, Lin H, Wang G (1996) On the bilayer phase transition temperatures for monoenoic phosphatidylcholines and phosphatidylethanolamines and the interconversion between them. Arch Biochem Biophys 334:135-142

Hyslop PA, Morel B, Sauerheber RD (1990) Organization and interaction of cholesterol and phosphatidylcholine in model bilayer membranes. Biochemistry 29(4):1025-1038

Hyvönen MT, Kovanen PT (2005) Molecular dynamics simulations of unsaturated lipid bilayers: effects of varying the number of double bonds. Eur Biophys J 34:294-305

Ichimori H, Hata T, Matsuki H, Kaneshina S (1999) Effect of unsaturated acyl chains on the thermotropic and barotropic phase transitions of phospholipid bilayer membranes. Chem Phys Lipids 100:151-164

Inoue T, Kitahashi T, Nibu Y (1999) Phase behavior of hydrated bilayer of binary phospholipid mixtures composed of 1,2-distearoylphosphatidylcholine and 1-stearoyl-2-oleoylphosphatidylcholine or 1-oleoyl-2-stearoylphosphatidylcholine. Chem Phys Lipids 99(1):103-109

Jensen MØ, Mouritsen OG, Peters GH (2004) Simulations of a membrane-anchored peptide: structure, dynamics, and influence on bilayer properties. Biophys J 86:3556-3575

Jerabek H, Pabst G, Rappolt M, Stockner T (2010) Membrane-mediated effect on ion channels induced by the anesthetic drug ketamine. J Am Chem Soc 132(23):7990-7997

Kamp JAFOD, Kauerz MT, Deenen LLMV (1975) Action of pancreatic phospholipase $A_{2}$ on phosphatidylcholine bilayers in different physical states. Biochim Biophys Acta 406(2):169-177

Kaneshina S, Ichimori H, Hata T, Matsuki H (1998) Barotropic phase transitions of dioleoylphosphatidylcholine and stearoyl-oleoylphosphatidylcholine bilayer membranes. Biochim Biophys Acta 1374:1-8
Keough KMW (1986) Modifications of lipid structure and their influence on mesomorphism in model membranes: the influence of hydrocarbon chains. Biochem Cell Biol 64(1):44-49

Keough KMW, Giffin B, Matthews PLJ (1989) Phosphatidylcholinecholesterol interactions: bilayers of heteroacid lipids containing linoleate lose calorimetric transitions at low cholesterol concentration. Biochim Biophys Acta Biomembr 983(1):51-55

Keough KMW, Parsons CS (1990) Differential scanning calorimetry of dispersions of products of oxidation of 1-stearoyl2-linoleoyl-sn-glycero-3-phosphocholine. Biochem Cell Biol 68(1):300-307

Kirsch SA, Böckmann RA (2016) Membrane pore formation in atomistic and coarse-grained simulations. Biochim Biophys Acta 1858(10):2266-2277. doi:10.1016/j.bbamem.2015.12.031

Klose G, Köenig B, Paltauf F (1992) Sorption isotherms and swelling of POPC in H20 and 2H20. Chem Phys Lipids 61(3):265-270

Köckritz A, Martin A (2008) Oxidation of unsaturated fatty acid derivatives and vegetable oils. Eur J Lipid Sci Technol 110:812-824

Köenig BW, Dietrich U, Klose G (1997a) Hydration and structural properties of mixed lipid/surfactant model membranes. Langmuir 13(3):525-532

Köenig BW, Strey HH, Gawrisch K (1997b) Membrane lateral compressibility determined by NMR and X-ray diffraction: effect of acyc chain polyunsaturation. Biophys J 73(4):1954-1966

König B (1992) Hydration in Phospholipidmembranen. Ph.D. Thesis, University of Leipzig, Leipzig

Koubi L, Saiz L, Tarek M, Scharf D, Klein ML (2003) Influence of anesthetic and nonimmobilizer molecules on the physical properties of a polyunsaturated lipid bilayer. J Phys Chem B 107(51):14500-14508

Koynova R, Caffrey M (1998) Phases and phase transitions of the phosphatidylcholines. Biochim Biophys Acta 1376:91-145

Kruyff BD, Demel RA, Slotboom AJ, van Deenen LLM, Rosenthal AF (1973) The effect of the polar headgroup on the lipid-cholesterol interaction: monolayer and differential scanning calorimetry study. Biochim Biophys Acta 307(1):1-19

Kučerka N, Tristram-Nagle S, Nagle JF (2005) Structure of fully hydrated fluid phase lipid bilayers with monounsaturated chains. J Membr Biol 208(3):193-202

Kučerka N, Nieh MP, Katsaras J (2011) Fluid phase lipid areas and bilayer thicknesses of commonly used phosphatidylcholines as a function of temperature. Biochim Biophys Acta 1808:27612771. doi:10.1016/j.bbamem.2011.07.022

Landau DP, Binder K (2009) A guide to Monte Carlo simulations in statistical physics. Cambridge University Press, Cambridge

Lantzsch G, Binder H, Heerklotz H (1994) Surface area per molecule in lipid/C12En membranes as seen by fluorescence resonance energy transfer. J Fluorescence 4(4):339-343

Lantzsch G, Binder H, Heerklotz H, Wendling M, Klose G (1996) Surface areas and packing constraints in POPC/C12EOn membranes. A time-resolved fluorescence study. Biophys Chem 58:289-302

Lavialle F, Levin IW (1980) Raman spectroscopic study of the interactions of dimyristoyl- and 1-palmitoyl-2-oleoylphosphatidylcholine liposomes with myelin proteolipid apoprotein. Biochemistry 19(26):6044-6050

Leach AR (2001) Molecular modelling. Principles and applications. Pearson Education Ltd, London

Leftin A, Molugu TR, Job C, Beyer K, Brown MF (2014) Area per lipid and cholesterol interactions in membranes from separated local-field ${ }^{13} \mathrm{C}$ NMR spectroscopy. Biophys J 107(11):2274-2286. doi:10.1016/j.bpj.2014.07.044

Litman BJ, Lewis EN, Levin IW (1991) Packing characteristics of highly unsaturated bilayer lipids: Raman spectroscopic 
studies of multilamellar phosphatidylcholine dispersions. Biochemistry 30(2):313-319

Lüscher-Mattli M (1989) Effect of the mitogenic lectin concanavalin A on the thermotropic behavior of glycosyl-free cationic lipids and their mixtures with zwitterionic lipids. Biopolymers 28(4):799-817

Lutterodt H, Luther M, Slavin M, Yin J, Parry J, Gao J, Yu L (2010) Fatty acid profile, thymoquinone content, oxidative stability, and antioxidant properties of cold-pressed black cumin seed oils. LWT Food Sci Technol 43:1409-1413

Lynch DV, Steponkus PL (1989) Lyotropic phase behavior of unsaturated phosphatidylcholine species: relevance to the mechanism of plasma membrane destabilization and freezing injury. Biochim Biophys Acta Biomembr 984(3):267-272

Lyubartsev AP, Rabinovich AL (2016) Force field development for lipid membrane simulations. Biochim Biophys Acta 1858(10):2483-2497. doi:10.1016/j.bbamem.2015.12.033

Lyubartsev AP, Laaksonen A (2000) A general and scalable parallel software package for arbitrary mixtures of molecules. Comput Phys Commun 128:565-589

Martyna GJ, Tuckerman ME, Tobias DJ, Klein ML (1996) Explicit reversible integrators for extended systems dynamics. Mol Phys 87(5):1117-1157

McCabe MA, Griffith GL, Ehringer WD, Stillwell W, Wassall SR (1994) ${ }^{2} \mathrm{H}$ NMR studies of isomeric $\omega 3$ and $\omega 6$ polyunsaturated phospholipid membranes. Biochemistry 33(23):7203-7210

McCusker MM, Grant-Kels JM (2010) Healing fats of the skin: the structural and immunologic roles of the $\omega-6$ and $\omega-3$ fatty acids. Clin Dermatol 28:440-451

Minkler PE, Hoppel CL (2010) Separation and characterization of cardiolipin molecular species by reverse-phase ion pair highperformance liquid chromatography-mass spectrometry. J Lipid Res 51:856-865

Molfino A, Amabile M, Monti M, Arcieri S, Fanelli F, Muscaritoli M (2016) The role of docosahexaenoic acid (DHA) in the control of obesity and metabolic derangements in breast cancer. Int J Mol Sci 17(4):505. doi:10.3390/ijms17040505

Mouritsen OG, Bagatolli LA (2016) Life-as a matter of fat: lipids in a membrane biophysics perspective (The Frontiers Collection). Springer, New York, Heidelberg, Dordrecht, London

Nabavi S, Bilotto S, Russo G, Orhan I, Habtemariam S, Daglia M, Devi K, Loizzo M, Tundis R, Nabavi S (2015) Omega-3 polyunsaturated fatty acids and cancer: lessons learned from clinical trials. Cancer Metastasis Rev 34(3):359-380. doi:10.1007/ s10555-015-9572-2

Napolitano GE, Heras H, Stewart AJ (1995) Fatty acid composition of freshwater phytoplankton during a red tide event. Biochem Syst Ecol 23(1):65-69

Nelson DL, Cox MM (2008) Lehninger principles of biochemistry, 5th edn. Freeman W.H. and Co, New York

Niebylski CD Jr, Salem N (1994) A calorimetric investigation of a series of mixed-chain polyunsaturated phosphatidylcholines: effect of $s n-2$ chain length and degree of unsaturation. Biophys J 67(6):2387-2393

Pabst G, Rappolt M, Amenitsch H, Laggner P (2000) Structural information from multilamellar liposomes at full hydration: full $q$-range fitting with high quality X-ray data. Phys Rev E 62:4000-4009

Pan J, Tristram-Nagle S, Nagle JF (2009) Effect of cholesterol on structural and mechanical properties of membranes depends on lipid chain saturation. Phys Rev E 80(021):931

Pasenkiewicz-Gierula M, Baczynski K, Markiewicz M, Murzyn K (2016) Computer modelling studies of the bilayer/ water interface. Biochim Biophys Acta 1858(10):2305-2321. doi:10.1016/j.bbamem.2016.01.024

Perly B, Smith ICP, Jarrell HC (1985) Effects of the replacement of a double bond by a cyclopropane ring in phosphatidylethanolamines: a ${ }^{2} \mathrm{H}$ NMR study of phase transitions and molecular organization. Biochemistry 24(4):1055-1063

Phillips MC, Hauser H, Paltauf F (1972) The inter- and intra-molecular mixing of hydrocarbon chains in lecithin/water systems. Chem Phys Lipids 8(1):127-133

Pinzi S, Garcia IL, Lopez-Gimenez FJ, de Castro MDL, Dorado G, Dorado MP (2009) The ideal vegetable oil-based biodiesel composition: a review of social, economical and technical implications. Energy Fuels 23:2325-2341

Pluhackova K, Böckmann RA (2015) Biomembranes in atomistic and coarse-grained simulations. J Phys Condens Matter 27:323103. doi:10.1088/0953-8984/27/32/323103

Pöyry S, Vattulainen I (2016) Role of charged lipids in membrane structures-insight given by simulations. Biochim Biophys Acta 1858(10):2322-2333. doi:10.1016/j.bbamem.2016.03.016

Rabinovich AL, Ripatti PO, Balabaev N (1999a) Molecular dynamics simulations of unsaturated lipid in monolayers: an investigation of bond ordering. Proc SPIE 3687:175-181

Rabinovich AL, Ripatti PO, Balabaev NK (1999b) Molecular dynamics investigation of bond ordering of unsaturated lipids in monolayers. J Biol Phys 25(2):245-262

Rabinovich AL, Ripatti PO, Balabaev N (2000) Molecular dynamics investigation of polar diacylglycerolipid monolayers: bond ordering properties. Proc SPIE 4064:144-155

Rabinovich AL, Ripatti PO, Balabaev NK, Leermakers FAM (2003) Molecular dynamics simulations of hydrated unsaturated lipid bilayers in the liquid-crystal phase and comparison to self-consistent field modeling. Phys Rev E 67(1):011909

Rabinovich AL, Balabaev NK (2001) Molecular dynamics simulations of unsaturated lipid bilayers. Proc SPIE 4348:215-224

Rabinovich AL, Lyubartsev AP (2013) Computer simulation of lipid membranes: methodology and achievements. Polym Sci Ser C 55:162-180

Rabinovich AL, Lyubartsev AP (2014) Bond orientation properties in lipid molecules of membranes: molecular dynamics simulations. J Phys Conf Ser 510:012022. doi:10.1088/1742-6596/510/1/012022

Rabinovich AL, Ripatti PO (1999) Intramolecular ordering of bonds in the cis-4,7,10,13,16,19-docosahexaenic chain. Dokl Biophys 364-366:6-9

Rabinovich AL, Ripatti PO (2000) A computer investigation of intramolecular bond ordering: unsaturated chains of natural lipids. Membr Cell Biol 13(5):697-714

Rand RP, Fuller N, Parsegian VA, Rau DC (1988) Variation in hydration forces between neutral phospholipid bilayers: evidence for hydration attraction. Biochemistry 27(20):7711-7722

Rand RP, Parsegian VA (1989) Hydration forces between phospholipid bilayers. Biochim Biophys Acta 988:351-376

Rincón-Cervera MÁ, Guil-Guerrero JL (2010) Preparation of stearidonic acid-enriched triacylglycerols from Echium plantagineum seed oil. Eur J Lipid Sci Technol 112:227-232

Rovere MTL, Christensen JH (2015) The autonomic nervous system and cardiovascular disease: role of n-3 PUFAs. Vascul Pharmacol 71:1-10. doi:10.1016/j.vph.2014.12.005

Rueter K, Haynes A, Prescott S (2015) Developing primary intervention strategies to prevent allergic disease. Curr Allergy Asthma Rep 15(7):40. doi:10.1007/s11882-015-0537-x

Sahena F, Zaidul ISM, Jinap S, Saari N, Jahurul HA, Abbas KA Norulaini NA (2009) PUFAs in fish: extraction, fractionation, importance in health. Compr Rev Food Sci Food Saf 8:59-74 
Sakuradani E (2010) Advances in the production of various polyunsaturated fatty acids through oleaginous fungus Mortierella alpine breeding. Biosci Biotechnol Biochem 74:908-917

Sánchez-Migallón MP, Aranda FJ, Gómez-Fernández JC (1996) Interaction between $\alpha$-tocopherol and heteroacid phosphatidylcholines with different amounts of unsaturation. Biochim Biophys Acta 1279:251-258

Santaren JF, Rico M, Guilleme J, Ribera A (1982) Thermal and ${ }^{13}$ C NMR study of dynamic structure of 1-palmitoyl-2-oleyl-snglycero-3-phosphocholine and 1-oleyl-2-palmitoyl-sn-glycero3 -phosphocholine in aqueous dispersions. Biochim Biophys Acta 687:231-237

Satoh A (2011) Introduction to practice of molecular simulation: molecular dynamics, Monte Carlo, Brownian dynamics, Lattice Boltzmann, dissipative particle dynamics. Elsevier, Amsterdam

Seelig J, Waespe-Sarcevic N (1978) Molecular order in cis and trans unsaturated phospholipid bilayers. Biochemistry 17(16):3310-3315

Separovich F, Gawrisch K (1996) Effect of unsaturation on the chain order of phosphatidylcholines in a dioleoylphosphatidylethanolamine matrix. Biophys J 71:274-282

Singh SP, Zhou X, Liu Q, Stymne S, Green AG (2005) Metabolic engineering of new fatty acids in plants. Curr Opin Plant Biol 8:197-203

Siu SWI, Vácha R, Jungwirth P, Böckmann RA (2008) Biomolecular simulations of membranes: physical properties from different force fields. J Chem Phys 128(125): 103

Smaby JM, Momsen MM, Brockman HL, Brown RE (1997) Phosphatidylcholine acyl unsaturation modulates the decrease in interfacial elasticity induced by cholesterol. Biophys $\mathbf{J}$ 73:1492-1505

Sonne J, Jensen MØ, Hansen FY, Hemmingsen L, Peters GH (2007) Reparametrization of all-atom dipalmitoylphosphatidylcholine lipid parameters enables simulation of fluid bilayers at zero tension. Biophys J 92:4157-4167

Sperling LS, Nelson JR (2016) History and future of omega-3 fatty acids in cardiovascular disease. Curr Med Res Opin 32(2):301311. doi:10.1185/03007995.2015.1120190

Stillwell W, Jenski LJ, Zerouga M, Dumaual AC (2000) Detection of lipid domains in docosahexaenoic acid-rich bilayers by acyl chain-specific fret probes. Chem Phys Lipids 104:113-132

Surewicz WK, Epand RM (1986) Phospholipid structure determines the effects of peptides on membranes. Differential scanning calorimetry studies with pentagastrin-related peptides. Biochim Biophys Acta 856(2):290-300
Swaney JB (1980) Mechanisms of protein-lipid interaction. Association of apolipoproteins A-I and A-II with binary phospholipid mixtures. J Biol Chem 255(18):8791-8797

Tada K, Miyazaki E, Goto M, Tamai N, Matsuki H, Kaneshina S (2009) Barotropic and thermotropic bilayer phase behavior of positional isomers of unsaturated mixed-chain phosphatidylcholines. Biochim Biophys Acta 1788:1056-1063

Tada K, Goto M, Tamai N, Matsuki H, Kaneshina S (2010) Pressure effect on the bilayer phase transition of asymmetric lipids with an unsaturated acyl chain. Ann N Y Acad Sci 1189:77-85

Toukan K, Rahman A (1985) Molecular dynamics study of atomic motions in water. Phys Rev B 31:2643-2648

Tuckerman M, Berne BJ, Martyna GJ (1992) Reversible multiple time scale molecular dynamics. J Chem Phys 97:1990-2001

Vilchéze C, McMullen TPW, McElhaney RN, Bittman R (1996) The effect of side-chain analogues of cholesterol on the thermotropic phase behavior of 1-stearoyl-2-oleoylphosphatidylcholine bilayers: a differential scanning calorimetric study. Biochim Biophys Acta 1279:235-242

Vincent M, Gallay J, Bony JD, Tocanne J (1985) Steady-state and time-resolved fluorescence anisotropy study of phospholipid molecular motion in the gel phase using 1-palmitoyl-2-[9(2-anthryl)-nonanoyl]-sn-glycero-3-phosphocholine as probe. Eur J Biochem 150:341-347

Wang G, Lin H, Li S, Huang C (1995a) Phosphatidylcholines with sn -1 saturated and $s n-2$ cis-monounsaturated acyl chains. J Biol Chem 270(39):22738-22746

Wang Z, Lin H, Li S, Huang C (1995b) Phase transition behavior and molecular structures of monounsaturated phosphatidylcholines. J Biol Chem 270(5):2014-2023

Wang X, Chan CB (2015) n-3 polyunsaturated fatty acids and insulin secretion. J Endocrinol 224(3):R97-R106. doi:10.1530/ JOE-14-0581

Winter R, Pilgrim WC (1989) A SANS study of high pressure phase transitions in model biomembranes. Ber Bunsen Ges Phys Chem 93(6):708-717

Wittkop B, Snowdon RJ, Friedt W (2009) Status and perspectives of breeding for enhanced yield and quality of oilseed crops for Europe. Euphytica 170:131-140

Yoon DY, Flory PJ (1978) Small angle neutron scattering by n-alkane chains. J Chem Phys 69(6):2536-2538

Zhurkin DV, Rabinovich AL (2015) Monte carlo properties of the hydrocarbon chains of phospholipid molecules. Russ J Phys Chem A 89(2):242-249. doi:10.1134/S0036024415020387 